\title{
Applications of Velocity-Stack \\ Methods to Seismic Data Processing
}

\section{U.S. GEOLOGICAL SURVEY BULLETIN 2102}

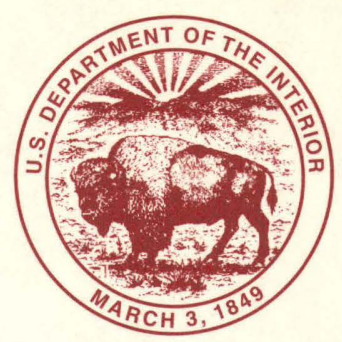




\section{AVAILABILITY OF BOOKS AND MAPS OF THE U.S. GEOLOGICAL SURVEY}

Instructions on ordering publications of the U.S. Geological Survey, along with prices of the last offerings, are given in the current-year issues of the monthly catalog "New Publications of the U.S. Geological Survey." Prices of available U.S. Geological Survey publications released prior to the current year are listed in the most recent annual "Price and Availability List." Publications that may be listed in various U.S. Geological Survey catalogs (see back inside cover) but not listed in the most recent annual "Price and Availability List" may no longer be available.

Reports released through the NTIS may be obtained by writing to the National Technical Information Service, U.S. Department of Commerce, Springfield, VA 22161; please include NTIS report number with inquiry.

Order U.S. Geological Survey publications by mail or over the counter from the offices listed below.

\section{BY MAIL}

\section{Books}

Professional Papers, Bulletins, Water-Supply Papers, Techniques of Water-Resources Investigations, Circulars, publications of general interest (such as leaflets, pamphlets, booklets), single copies of Earthquakes \& Volcanoes, Preliminary Determination of Epicenters, and some miscellaneous reports, including some of the. foregoing series that have gone out of print at the Superintendent of Documents, are obtainable by mail from

\section{U.S. Geological Survey, Map Distribution Box 25286, MS 306, Federal Center Denver, CO 80225}

Subscriptions to periodicals (Earthquakes \& Volcanoes and Preliminary Determination of Epicenters) can be obtained ONLY from the

\section{Superintendent of Documents Government Printing Office Washington, DC 20402}

(Check or money order must be payable to Superintendent of Documents.)

\section{Maps}

For maps, address mail orders to

\section{U. S. Geological Survey, Map Distribution Box 25286, Bldg. 810, Federal Center Denver, CO 80225}

Residents of Alaska may order maps from

\author{
U.S. Geological Survey, Earth Science Information Center \\ 101 Twelfth Ave., Box 12 \\ Fairbanks, AK 99701
}

\section{OVER THE COUNTER}

\section{Books and Maps}

Books and maps of the U.S. Geological Survey are available over the counter at the following U.S. Geological Survey offices, all of which are authorized agents of the Superintendent of Documents.

- ANCHORAGE, Alaska-Rm. 101, 4230 University Dr.

- LAKEWOOD, Colorado-Federal Center, Bldg. 810

- MENLO PARK, California-Bldg. 3, Rm. 3128, 345 Middlefield Rd.

- RESTON, Virginia-USGS National Center, Rm. 1C402, 12201 Sunrise Valley Dr.

- SALT LAKE CITY, Utah-Federal Bldg., Rm. 8105, 125 South State St.

- SPOKANE, Washington-U.S. Post Office Bldg., Rm. 135, West 904 Riverside Ave.

- WASHINGTON, D.C.-Main Interior Bldg., Rm. 2650, 18th and C Sts., NW.

\section{Maps Only}

Maps may be purchased over the counter at the following U.S. Geological Survey offices:

- FAIRBANKS, Alaska-New Federal Bldg, 101 Twelfth Ave.

- ROLLA, Missouri-1400 Independence Rd.

- STENNIS SPACE CENTER, Mississippi-Bldg. 3101 


\section{Applications of Velocity-Stack Methods to Seismic Data Processing}

By Myung W. Lee and Warren F. Agena

U.S. GEOLOGICAL SURVEY BULLETIN 2102

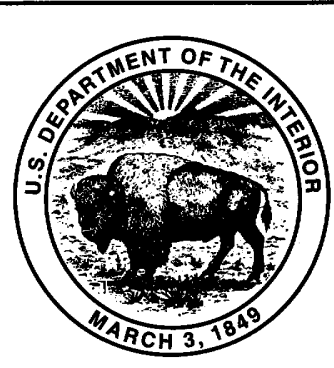

UNITED STATES GOVERNMENT PRINTING OFFICE, WASHINGTON : 1994 


\title{
U.S. DEPARTMENT OF THE INTERIOR BRUCE BABBITT, Secretary
}

\author{
U.S. GEOLOGICAL SURVEY \\ Gordon P. Eaton, Director
}
For sale by U.S. Geological Survey, Map Distribution
Box 25286, MS 306, Federal Center
Denver, CO 80225

\begin{abstract}
Any use of trade, product, or firm names in this publication is for descriptive purposes only and does not imply endorsement by the U.S. Government
\end{abstract}

\section{Library of Congress Cataloging-in-Publication Data}

Lee, Myung W.

Applications of velocity-stack methods to seismic data processing

by Myung W. Lee and Warren F. Agena.

p. cm.-(U.S. Geological Survey bulletin : 2102)

Includes bibliographical references.

Supt. of Docs. no. : I 19.3:2102

1. Seismology-Mathematical models-Data processing. I. Agena, W. F.

II. Title. III. Title: Velocity-stack methods. IV. Series.

QE75.B9 no. 2102

[QE539.2.D36]

$557.3 \mathrm{~s}-\mathrm{dc} 20$

[551.2'2'015118] 


\section{CONTENTS}

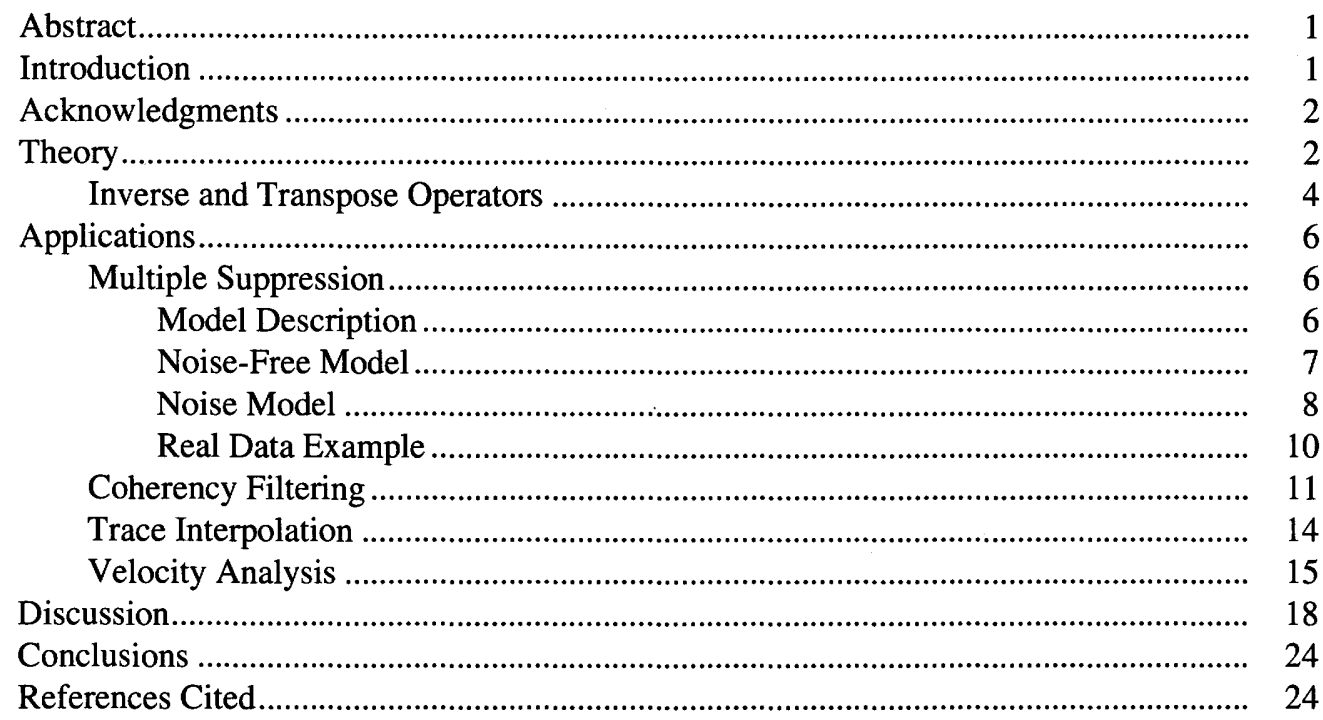

\section{FIGURES}

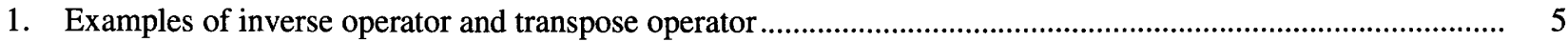

2. Response of inverse operator and transpose operator using parabolic moveout..................................................... 6

3. Example of performance of inverse and transpose operator ......................................................................... 7

4. Multiple and primary models of synthetic model filtered with a symmetrical wavelet ......................................... 8

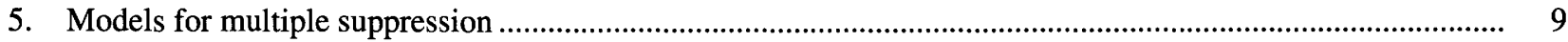

6. Multiple suppression for three multiple models with and without static shifts................................................ 10

7. Amplitude analysis of multiple suppression for model 2 ................................................................... 11

8. Multiple suppression for model 3 using an inverse operator ................................................................. 12

9-10. Multiple suppression for model 1 with random-amplitude noise and static shifts using:

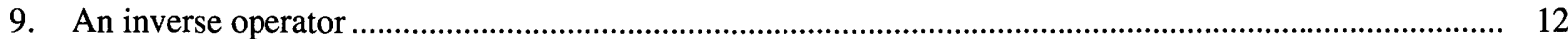

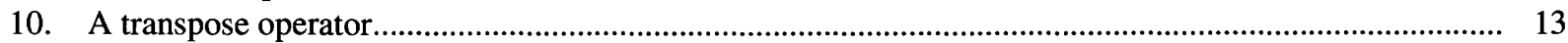

11. Straight stacked section of a profile acquired at Lake Baikal ...............................................................

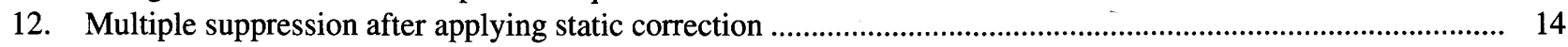

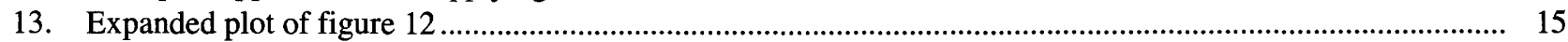

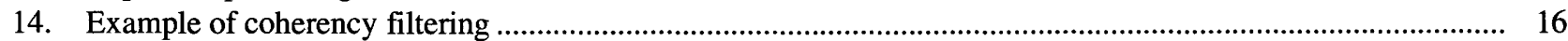

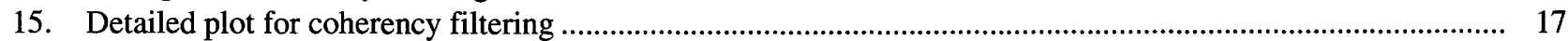

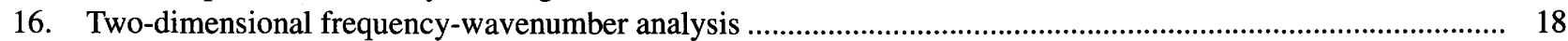

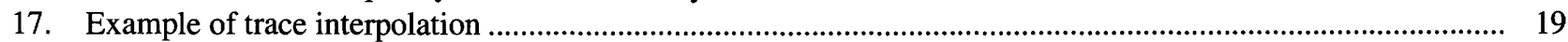

18-19. Examples of velocity analysis using the:

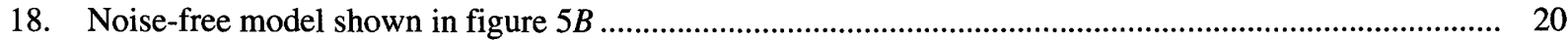

19. Noise model with random-amplitude noise and static shifts......................................................... 21

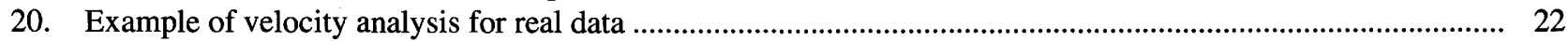

21. Example of multiple suppression with and without static correction............................................................. 23

22. Effect of random noise and static correction in velocity analysis ................................................................... 24 



\title{
APPLICATIONS OF VELOCITY-STACK METHODS TO SEISMIC DATA PROCESSING
}

\author{
By Myung W. Lee and Warren F. Agena
}

\begin{abstract}
A velocity stack is originally defined as the summation of a seismic event along a hyperbolic moveout trajectory, but this definition is extended to include both linear and parabolic moveouts in this report. Thus, our velocity stack can be used for modeling events with a variety of moveouts. The ability to model via a velocity stack provides us a number of applications in the area of seismic data processing, such as multiple suppression, coherency filtering, trace interpolation, and velocity analysis. To take advantage of the computational efficiency in the frequency domain, only linear and parabolic moveouts are considered here.

Velocity stacking can be accomplished through the use of either a transpose operator, such as in conventional velocity analysis, or by an inverse operator. These operators depend only on the source-receiver offsets of seismic traces and the range of velocities. The choice between the transpose and the inverse operator for computational purposes is immaterial because either operator need only be computed once. Some advantages in using the inverse operator over the transpose operator include preserving the input waveform (the transpose operator tends to bias the frequency content toward lower frequencies) and minimizing any mixing effects. These favorable properties of the inverse operator also allow for better coherency filtering and trace interpolation.

Because velocity stacking works well at all sourcereceiver offsets, its performance in suppressing multiples is better than two-dimensional filtering in the frequency-wavenumber domain. When working with good-quality seismic data, using an inverse operator for velocity analysis has significant advantages over a transpose operator. However, as the quality of the data decreases with the addition of more noise, the use of the inverse operator is only marginally better.
\end{abstract}

\section{INTRODUCTION}

Normal moveout (NMO) correction and stacking are standard, industry, seismic-data-processing sequences. Combining these two processes is identical to stacking along a family of hyperbolic curves and is described as a velocity stack using a transpose operator (Thorson, 1984; Thorson and Claerbout, 1985). Conventional constant velocity analysis is one form of velocity stack using a transpose operator. It is a linear transformation and maps the data into velocity space. One problem in using a transpose operator is the smearing effect it has on the data. To better discriminate signal and noise in velocity space, Thorson (1984) proposed an inverse operator, which essentially deconvolves the spatial mixing effect of the transpose operator.

Thorson (1984) described two approaches in estimating an inverse operator: the generalized inverse and stochastic inverse. The original formulation of Thorson (1984) requires inversion of a large matrix. Hampson (1986) showed that, for a typical 96-trace seismic shot of 3-s duration, the matrix order would be 150,000 by 150,000 . Thorson and Claerbout (1985) suggested an iterative solution using a conjugate-gradient method, which is still time consuming but more efficient. In order to reduce computational time in estimating an inverse operator further, Hampson (1986) proposed a parabolic trajectory rather than a hyperbolic trajectory. The parabolic trajectory is not a physically observable traveltime curve in the offset domain, but it can be approximated by using NMO corrections with appropriate velocities. This approximation enables the use of the Fourier transform, and a computationally efficient method can be derived.

Computing an inverse operator is more time consuming than computing a transpose operator. However, because both computations depend only on offset and number of velocities (or slownesses), these operators need only be computed once, and, hence, the choice between the two methods based on computing time alone is immaterial.

To a first-order approximation, seismic data in the offset domain can be considered as a linear superposition of many events whose traveltimes describe certain trajectories. The collection of events are defined as a model in the velocity domain. The inverse operator allows us to accurately estimate such a model from seismic data contaminated with random noise, and it provides us with a number of processing techniques, such as multiple removal, coherency filtering, trace interpolation, and velocity analysis. 
The performances of both the inverse and transpose operators for each application are compared, and the advantages of the inverse operator are discussed.

Thorson (1984) described multiple suppression by stacking along the primary velocity in velocity space. He utilized the property of better velocity discrimination of an inverse operator in his multiple suppression. Another approach to multiple suppression is to model the multiple energy by an inverse operator and subtract this multiple energy from the original data. This approach of multiple suppression by velocity stack is described well by Hampson (1986), and the actual performance for a field data set is documented by Geist and others (1990). This paper employs Hampson's approach and uses models to quantify the performance of multiple suppression-we emphasize the use of static corrections for better multiple elimination.

The random noise in seismic data cannot be modeled as coherent events using a velocity stack. By transforming the data into model space and back to the offset domain, random noise will be reduced. This property can be used as a coherency-filtering or a signal-enhancement technique. Kong and others (1985) and Monk (1992) described nonlinear, coherency-filtering techniques using the invertible Radon transform or $\tau-p$ transform. Seismic events can also be predicted along various moveouts in model space, and this property can be used in trace interpolation. An inverse operator used for coherency filtering and trace interpolation produces data with reduced trace mixing (smearing) and preserves the high-frequency components.

The compact response, or reduced side lobes, of an inverse operator in the slowness domain is ideally suited for high-resolution velocity analysis (Thorson, 1984). However, noise introduced into real data sets degrades the performance of an inverse operator. This limitation in the presence of noisy data will be discussed later.

\section{ACKNOWLEDGMENTS}

We thank John J. Miller and William J. Stephenson for constructive reviews. All data were processed using CogniSeis DISCO software with additional programs developed by U.S. Geological Survey personnel.

\section{THEORY}

Detailed theoretical development of inverse velocity stacking can be found in Thorson (1984) and Hampson (1986). To a first-order approximation, considering only arrival times, seismic data can be modeled as a superposition of laterally coherent events. For example, a plane-wave response of dipping layers consists of laterally coherent events whose stepout times are linear with distance. Also, a seismic event recorded on a horizontal-receiver spread from a shot can be modeled as an event whose traveltime is represented by a hyperbolic curve. The only difference between the above two examples is the functional relationship of arrival times with respect to the appropriate coordinate system. In the data space or offset space, we use offset, $h$, and two-way time, $t$, as variables, and in the model space or velocity space we use slowness, $p$, and zero-offset time, $\tau$. Therefore, the seismic data $d(h, t)$ can be represented by:

$$
d(h, t)=\iint u(p, \tau) \delta(f(t, h, \tau, p)) d p d \tau+n(h, t)
$$

where

$d(h, t)$ is the measured seismogram at offset, $h$, and twoway time, $t$,

$u(p, \tau)$ are seismic models with slowness, $p$, and zero-offset time, $\tau$,

$\delta$ is the Dirac delta function,

$f(t, h, \tau, p)$ is the traveltime relationship between data and model spaces, and

$n(h, t)$ is the measurement noise at offset, $h$, and two-way time, $t$.

Three functional relationships, $f(t, h, \tau, p)$, in equation $1 \mathrm{a}$ are considered by numerous authors. Stoffa and others (1981) used $f(t, h, \tau, p)=\tau-(t-p h)$ as their functional relationship (this is the well-known slant stack or $\tau-p$ operation), whereas Thorson (1984) used a hyperbolic relationship, $f(t, h, \tau, p)=\tau-\sqrt{t^{2}-p^{2} h^{2}}$, for his inverse velocity stack. Hampson (1986) used a parabolic approximation to a hyperbolic traveltime curve (which is $f(t, h, \tau, p)=\tau-\left(t-s h^{2}\right)$, with a new parameter, $s$ ) in his study of inverse velocity stack for multiple removal. The meaning of $s$ will be discussed later. In this section, it is treated as a parameter similar to slowness, $p$.

Thorson (1984) defined the integral relationship in equation 1a without a noise term as an operator, $L$, such that $d(h, t)=L u(p, \tau)$ and defined a transpose operator, $L^{T}$, as $u(p, \tau)=L^{T} d(h, t)$. Or:

$$
\begin{gathered}
u(p, \tau)=L^{T} d(h, t)= \\
\iint d(h, t) \delta(f(t, h, \tau, p)) d h d t
\end{gathered}
$$

In the operator $L$, the variable $\tau$ is expressed as a function of $t$, and in the operator $L^{T}$, the variable $t$ is expressed as a function of $\tau$. Equation $1 \mathrm{~b}$ indicates that performing normal moveout correction and stacking, or stacking along the moveout defined by $f(t, h, \tau, p)$, are methods of transforming data into velocity space or model space. Under what conditions is this operation invertible? In other words, what operator will correctly invert the velocity space into the data space? As pointed out by Thorson (1984), the transpose operator defined in equation $1 \mathrm{~b}$ does not work well for a truncated data set, such as any real data set. The purpose of this section is to derive an efficient inverse operator that performs better than the transpose operator defined in equation $1 b$. 
A computationally efficient inverse operator is difficult to derive if a hyperbolic moveout is assumed in equation 1b. But, for a linear moveout or parabolic moveout, an efficient method can be derived in the frequency domain (Hampson, 1986). If we use a parabolic relationship for $f$ in equation 1a, then:

$$
\begin{gathered}
d(h, t)=\iint u(s, \tau) \delta\left(\tau-\left(t-s h^{2}\right)\right) d s d \tau+n(h, t) \\
=\int u\left(s, t-s h^{2}\right) d s+n(h, t)
\end{gathered}
$$

Equation 2 indicates that the seismic data can be modeled by summing the model along a parabolic trajectory. Equation 2 also indicates that the data space can be modeled by a convolution of the model $u(p, \tau)$ with the parabolic trajectory in the time domain. This convolutional property using the parabolic or linear traveltime curve in equation 1 is useful in the frequency domain because convolution in the time domain can be represented by multiplication in the frequency domain.
Let us take a Fourier transform of equation 2, and let upper-case variables represent the Fourier-transformed variables. Then:

$$
\begin{gathered}
\int d(h, t) e^{-i \omega t} d t= \\
\iiint u(s, \tau) \delta\left(\tau-\left(t-s h^{2}\right)\right) e^{-i \omega t} d s d \tau d t+\int n(h, t) e^{-i \omega t} d t \\
D(h, \omega)=\iint u(s, \tau) e^{-i \omega \tau} e^{-i \omega s h^{2}} d s d \tau+N(h, \omega) \\
=\int U(s, \omega) e^{-i \omega s h^{2}} d s+N(h, \omega)
\end{gathered}
$$

Equation (3) is valid for a continuous variable in $h$ and $s$. Let us assume that the offset, $h$, has only discrete values $h_{1}, h_{2}, \ldots, h_{N}$, and $s$ has discrete values $s_{1}, s_{2}, \ldots, s_{M}$. Then equation 3 can be written, using a matrix notation (a matrix is denoted as a bold-faced, upper-case letter) as:

$$
\mathbf{D}=\mathbf{L} \mathbf{U}+\mathbf{N}
$$

where

D is a column vector of $\left(D\left(h_{1}, \omega\right), D\left(h_{2}, \omega\right), \ldots, D\left(h_{N}, \omega\right)\right)$ with dimension $N$,

$\mathrm{U}$ is a column vector of $\left(U\left(s_{1}, \omega\right), U\left(s_{2}, \omega\right), \ldots, U\left(s_{M}, \omega\right)\right)$ with dimension $M$,

$\mathrm{N}$ is a column vector of $\left(N\left(h_{1}, \omega\right), N\left(h_{2}, \omega\right), \ldots, N\left(h_{N}, \omega\right)\right)$, and

$\mathbf{L}$ is an $N \times M$ matrix with element of $L_{n m}=e^{-i \omega s_{m} h^{2}{ }_{n}}$.

Explicitly, equation 4 can be written:

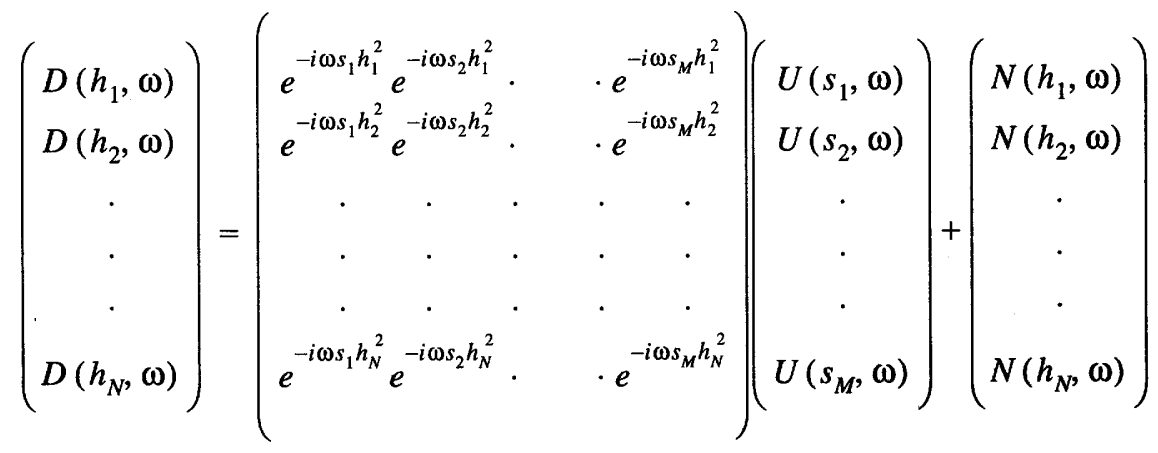


Now the question is how to compute model space, $\mathbf{U}$, from the noisy discrete data, $\mathbf{D}$, while minimizing the misfit function or noise, $\mathbf{N}$. Thorson and Claerbout (1985) demonstrated that this problem can be solved as an overdetermined linear system. The standard least-squares solution to equation 4 is:

$$
\mathbf{U}=\left(\mathbf{L}^{T} \mathbf{L}\right)^{-1} \mathbf{L}^{T} \mathbf{D} \equiv \mathbf{G D}
$$

Equation 6 shows the desirable inverse operator, $\mathbf{G}=$ $\left(\mathbf{L}^{T} \mathbf{L}\right)^{-1} \mathbf{L}^{T}$, in the velocity stack.

The meaning of the $s$ in the parabolic moveout is not as straightforward as the $p$ in the linear or hyperbolic moveout because this is not a physically observable moveout. The linear relationship between the time variables ( $\tau$ and $t$ ) makes possible the frequency domain operation in equation 1 . Under which circumstances can a hyperbolic moveout be approximated by a parabolic moveout that is linear in $\tau$ and $t$ ? The hyperbolic-moveout equation can be expanded using a Taylor series:

$$
\begin{gathered}
\sqrt{\tau^{2}+p^{2} h^{2}}=\tau \sqrt{1+\left(p^{2} h^{2} / \tau^{2}\right)} \\
=\tau\left(1+\frac{p^{2} h^{2}}{2 \tau^{2}}+\frac{p^{4} h^{4}}{8 \tau^{4}} \ldots . .\right)=\tau-\frac{p^{2} h^{2}}{2 \tau}-\frac{p^{4} h^{4}}{8 \tau^{3}} \ldots \ldots
\end{gathered}
$$

If $\left(p^{2} h^{2} / \tau^{2}\right)<<1$, dropping higher order terms can be justified. The only adjustable variable that will satisfy this condition is the slowness variable, $p$, and this adjustment can be accomplished by applying a normal moveout (NMO) correction with a slowness, $p_{c}$, as shown in Hampson (1986). Therefore the time $\left(t^{\prime}\right)$ after the NMO correction with $p_{c}$ can be written using equation 7 :

$$
\begin{gathered}
t^{\prime}=\tau+\sqrt{\tau^{2}+p^{2} h^{2}}-\sqrt{\tau^{2}+p_{c}^{2} h^{2}} \\
=\tau+\frac{h^{2}}{2 \tau}\left(p^{2}-p_{c}^{2}\right) \ldots \approx \tau+\frac{h^{2} p_{r}^{2}}{2 \tau} \ldots
\end{gathered}
$$

Therefore, if $p_{r}$ is very small or $p$ is close to $p_{c}$, a parabolic approximation can be justified. The parameter $s$ defined in equation 2 is $\left(p_{r}{ }^{2} / 2 \tau\right)$, and, strictly speaking, it is not a slowness parameter. Because the coefficient $h^{2}$ in the parabolic approximation is a function of zero-offset time, the shape of a parabola for a given $s$ is a function of zero-offset time, different from the slant stack. Also, smearing can be expected as events deviate from the ideal parabolic form.

\section{INVERSE AND TRANSPOSE OPERATORS}

Denoting the element of inverse operator $\mathbf{G}$ as $G_{i j}$, equation 6 can be written as:

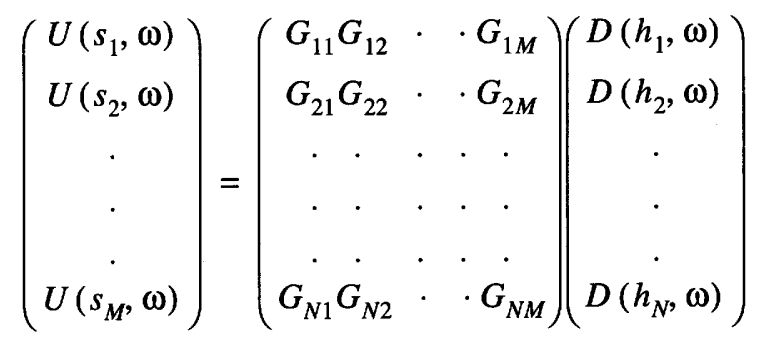

For a given frequency, each element of $\mathbf{G}$ is a complicated function of $h$ and $s$, which is easily seen from equation 5 , but does not involve actual data. In other words, for the same field geometry (same offset) and range of slownesses, the same inverse operator can be applied to the data set to compute $\mathbf{U}$ or $\mathbf{D}$. Therefore, we can pre-compute an inverse operator or a transpose operator before applying it to the data. Because we only have to compute the operator once, the fact that computing an inverse operator is more expensive that computing the transpose operator is rather trivial.

Figure $1 A$ shows the elements of an inverse operator, $G_{i j}$, convolved with a zero-phase band-pass wavelet for a parabolic moveout, and figure $1 B$ shows the transpose operator. The operators were computed with 12 offsets and nine slownesses. The near-offset and far-offset distances shown in figure 1 are $0 \mathrm{~m}$ and $275 \mathrm{~m}$, respectively, and the moveout time at the far offset ranges from $-60 \mathrm{~ms}$ to $60 \mathrm{~ms}$. As can be seen from figure 1 , the elements of the transpose operator is essentially a band-pass wavelet located at a trajectory of the parabolic moveout, whereas those of the inverse operator are a very complicated function of offset and slowness along the parabolic moveout trajectory. Notice the waveform changes, including polarity reversal, for the inverse operator. The waveforms of the transpose operator are identical for all offsets and slowness values, so the application of a transpose operator is the same as straight stacking along a parabolic moveout. The waveforms of the inverse operator are all different from each other, so the application of an inverse operator is similar to a weighted stack along a parabolic moveout.

If the numerical values of $\mathbf{D}$ in equation 9 are identical for all offsets, as for a horizontal event with the same wavelets, the model or velocity space can be derived by summing the rows of the inverse operator or summing along the offsets. Summing along the offsets is the same operation as summing each slowness panel in figure 1 . The output of model space for the operators shown in figure 1 is shown in figure $2 A$ for the inverse operator and is shown in figure $2 C$ for the transpose operator. The output of the inverse operator is symmetrical in time, but this is not true for the transpose operator. The amplitude of the inverse operator decays quickly with increasing moveout time, whereas the amplitude of the transpose operator decays slowly with increasing moveout time. The increased lateral spread of the transpose operator in the slowness domain causes lateral smearing in the velocity stack. 


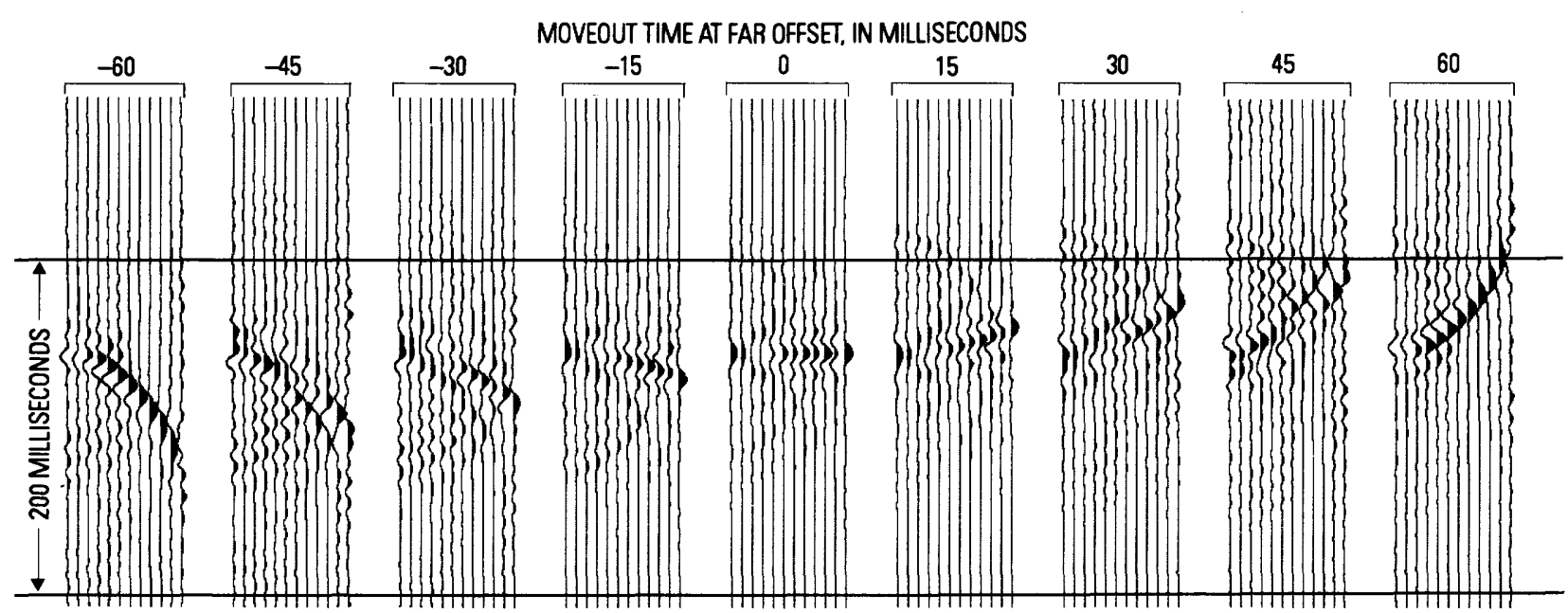

A

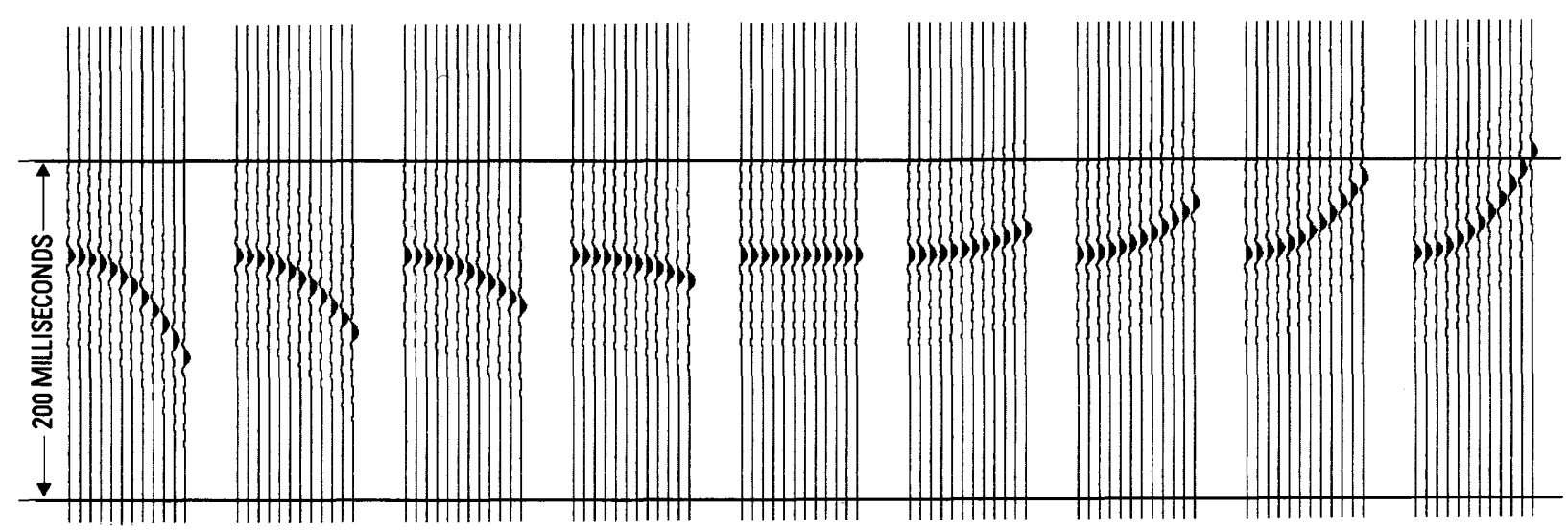

B

Figure 1. $A$, Inverse operator; $B$, Transpose operator convolved with a zero-phase band-pass wavelet of $4 / 6-85 / 100 \mathrm{~Hz}$ for a parabolic moveout with moveout time of $-60 \mathrm{~ms}$ to $60 \mathrm{~ms}$ at the far offset. For each moveout time at the far offset, 12 offsets are shown; these consist of, from left to right, the near offset (equal to $0 \mathrm{~m}$ ) to the far offset (equal to $275 \mathrm{~m}$ ).

The difference in performance between the inverse and the transpose operator can be easily examined by summing $\mathbf{U}$ over all slownesses. The result is shown in figure $2 B$ for the inverse operator and in figure $2 D$ for the transpose operator. Equation 3 indicates that integration with respect to slowness in the model space with $h=0$ will result in the zero-offset data in the offset domain. Therefore, if accurate $\mathbf{U}$ is modeled from $\mathbf{D}$ (data in the offset domain), a summation of the model $\mathbf{U}$ along the slowness will reproduce the input data. The result of this operation for the inverse operator, shown in figure $2 B$, clearly shows the waveform as identical to the input, which is a zero-phase band-pass wavelet with $4 / 6-85 / 100 \mathrm{~Hz}$, but the result of the transpose operator shows a bias toward lower frequency. This is one reason why the conventional velocity-analysis method, summing along the assumed velocity trajectory, shows a lack of resolution, as mentioned in Thorson (1984). For horizontal events, this result can be represented by:

$$
D(h=0, \omega)=\sum_{m=1}^{M} U\left(s_{m}, \omega\right)=\sum_{m=1}^{M} \sum_{n=1}^{N} G_{n m}
$$

This equation can be used to examine whether the computed inverse operator, $\mathbf{G}$, is correct or not.

An application of inverse and transpose operators in creating a model space is shown in figure 3 . Figure $3 A$ shows 

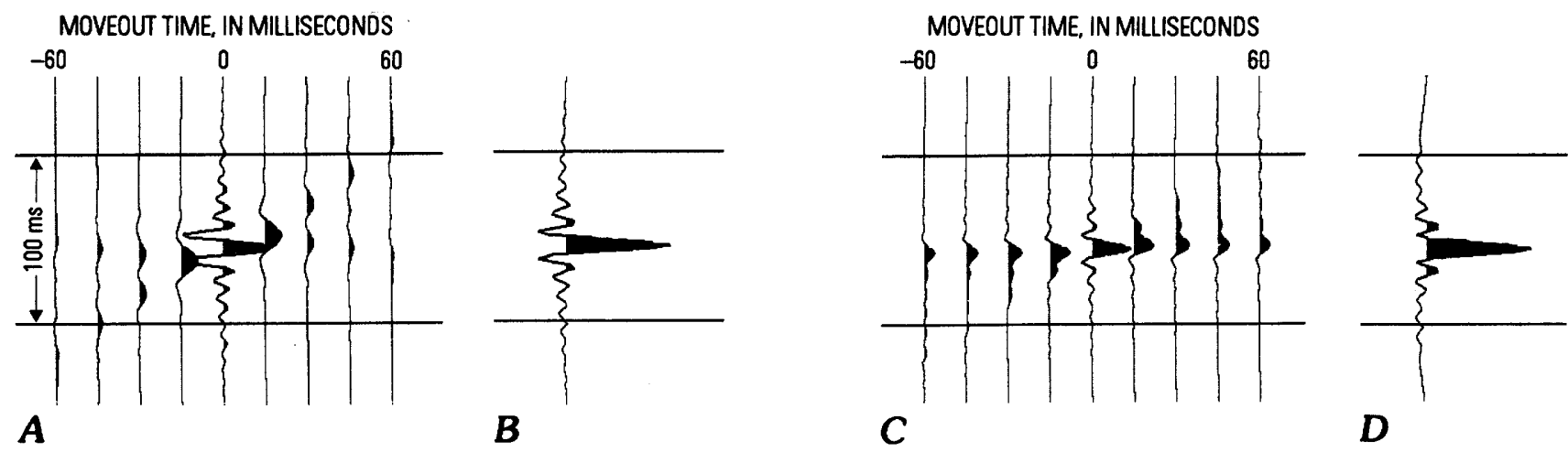

Figure 2. Response of inverse and transpose operator using parabolic moveout trajectory for a horizontal event. $A$, Response in slowness domain of inverse operator; $B$, Summation of response of inverse operator with respect to slowness; $C$, Response in slowness domain of transpose operator; $D$, Summation of response of transpose operator with respect to slowness. Note difference of the waveform in $B$ and $D$.

the noise-free input data; figure $3 B$ shows the modeled data using the inverse operator; and figure $3 C$ shows the result of applying the transpose operator. As indicated in figure 2 , the result of the transpose operator fails to model the higher frequency part of the input data. Loss of the high-frequency component is evident at the far offsets. Results from using an inverse operator, however, show an almost perfect reproduction of the input data.

\section{APPLICATIONS}

The ability to extract model space from a noisy data set can be used for a variety of seismic-data-processing techniques, and a number of applications of inverse velocity stack, such as velocity analysis, is discussed in Thorson (1984). Hampson (1986) presented an application to remove multiples using a parabolic approximation. In this paper, applications of the inverse and transpose operators will be discussed in the area of multiple suppression, coherency filtering, velocity analysis, and spatial interpolation.

\section{MULTIPLE SUPPRESSION}

The methodology of multiple suppression using the velocity stack can be summarized in the following five steps:

1. Apply NMO correction using stacking velocities of multiples.

2. Model the multiple event using the inverse or transpose operator.

3. Subtract the modeled multiple events in step 2 from the original data.

4. Remove the NMO correction applied in step 1.

5. Stack with the primary velocity.

The performance of multiple suppression outlined above is analyzed using models and a real data set.

\section{MODEL DESCRIPTION}

In order to analyze the performance of the multiple suppression technique by a velocity stack, a 24-channel common midpoint (CMP) gather, with near offset of $400 \mathrm{~m}$ and far offset of 2,700 m, was generated. Figure 4 shows the synthetic model filtered with a $4 / 6-42 / 54-\mathrm{Hz}$ symmetrical wavelet. Figure $4 A$ shows the multiple model, and figure $4 B$ shows a primary model. In figure $4 A$, the event near $2 \mathrm{~s}$ at the near offset represents a reference trace to measure the performance of the multiple suppression technique, and events below $2.5 \mathrm{~s}$ represent multiple energy. The average rootmean-square (RMS) amplitude of multiple energy is very close to the RMS amplitude of the reference trace. The RMS velocity of multiples is modeled by a linearly increasing function with depth: $1,400 \mathrm{~m} / \mathrm{s}$ at $2,500 \mathrm{~ms}$ and $1,600 \mathrm{~m} / \mathrm{s}$ at $4,500 \mathrm{~ms}$. Four primary events with identical amplitude and polarity are chosen and shown in figure $4 B$. The amplitude of the primaries in figure $4 B$ is 20 percent of the reference amplitude in figure $4 A$, and the locations and RMS velocities of four primaries in figure $4 B$ are: $3,000 \mathrm{~ms}$ with $1,600 \mathrm{~m} / \mathrm{s}$; $3,420 \mathrm{~ms}$ with $1,800 \mathrm{~m} / \mathrm{s} ; 3,800 \mathrm{~ms}$ with $2,000 \mathrm{~m} / \mathrm{s}$; and $3,950 \mathrm{~ms}$ with $2,200 \mathrm{~m} / \mathrm{s}$.

In our model study, figure $4 A$ was mixed with figure $4 B$ for a variety of scale factors. Three synthetic models were generated for the analysis.

Model 1: Multiple strength $14 \mathrm{~dB}$ higher than primaries.

Model 2: Multiple strength almost equal to primaries.

Model 3: Multiple strength $20 \mathrm{~dB}$ higher than primaries.

Figure $5 A$ shows model 1 , and figure $5 B$ shows model 2 . Notice that, in model 1 , primary events cannot be observed by the eye. The amplitude relationship in figure $5 A$ is similar to the field data example presented later. Figure $5 C$ is model 1 with random noise added and will be used later. 


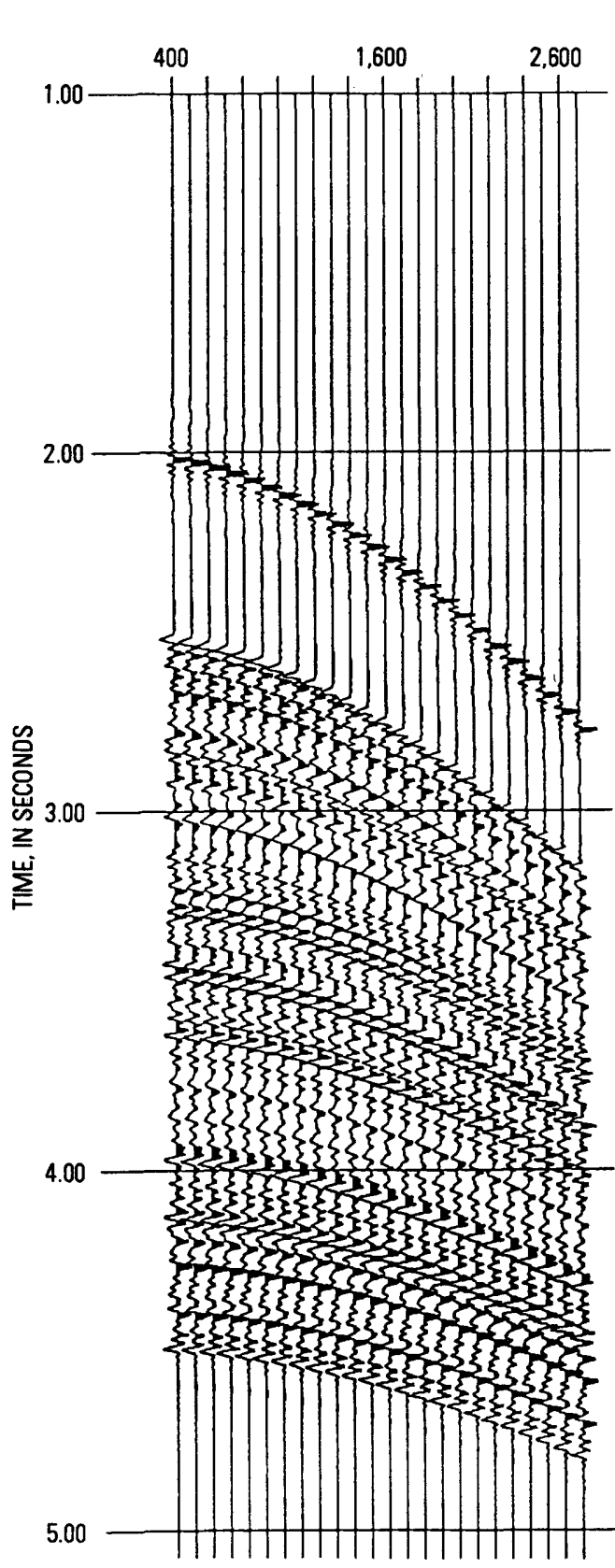

A

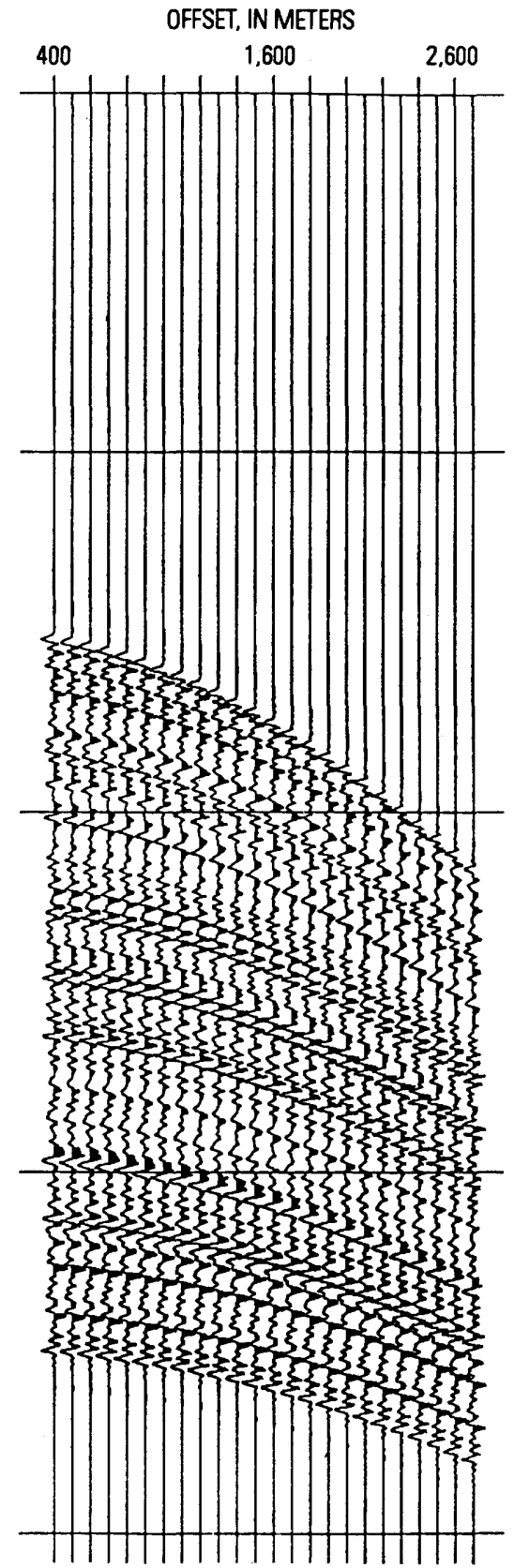

B
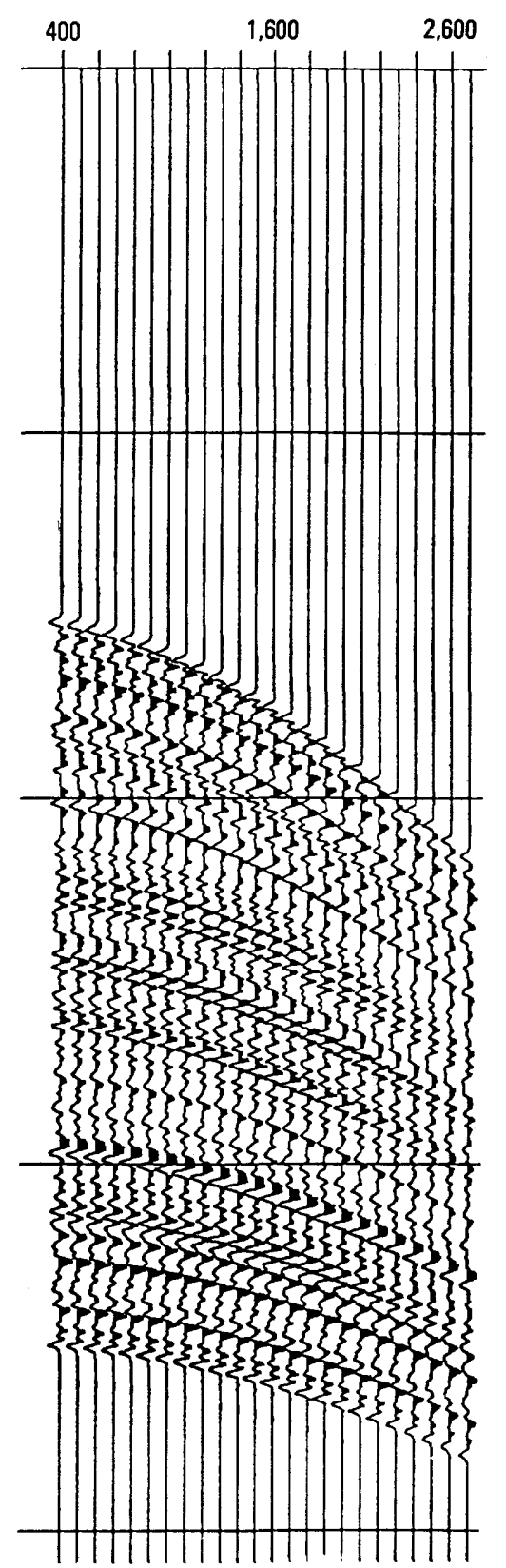

C

Figure 3. Example of performance of inverse and transpose operator. A, Original input; $B$, Modeling by an inverse operator; $C$, Modeling by a transpose operator.

\section{NOISE-FREE MODEL}

The results of straight stacking for noise-free models are shown in the "a" parts of figure 6 , and results of multiple suppression by a velocity stack using the inverse operator (inverse velocity stack) are shown in the "c" parts of figure 6. The results for the noise-free model (the "c" parts of fig. 6) indicate that multiple suppression by the inverse velocity stack suppresses the multiple energy enough to recognize the primaries. Also, the result shown for model 2, "a" part, indicates that straight stacking will be sufficient to suppress multiples when the multiple strength is almost the same as that of the primaries (model 2). However, it demonstrates that, if the multiple strength is more than $14 \mathrm{~dB}$ higher than the primaries, straight stacking is not enough to enhance the primaries (fig. 6, models 1 and 3, "a" parts), and some kind of prestack multiple-suppression technique, such as an inverse velocity stack, is required. 


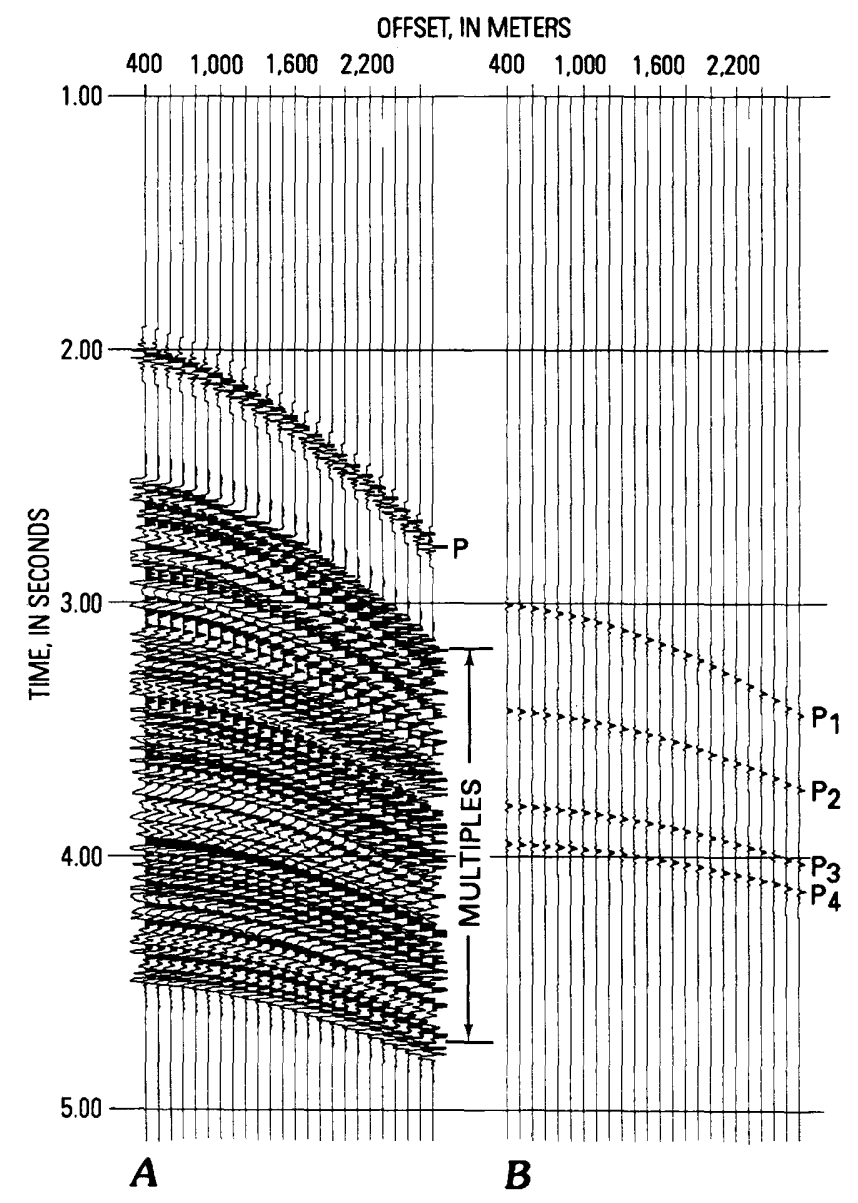

Figure 4. Synthetic model filtered with a 4/6-42/54-Hz symmetrical wavelet. $A$, Multiple model except the event at $2 \mathrm{~s}$, which serves as a reference amplitude; $B$, Primary model with four events. See text for additional explanation.

In order to see the details of multiple suppression, an amplitude analysis of the result of velocity stack for model 2 (fig. 6, model 2, "a" part) was performed, and the result is shown in figure $7 A$. This figure indicates that the velocity stack suppressed the multiple energy on the order of $40 \mathrm{~dB}$. Notice the five peaks near the $-10-\mathrm{dB}$ line in figure $7 \mathrm{~A}$, indicating almost perfect primary recovery. On the other hand, a straight stack only suppress the multiple energy on the order of $12 \mathrm{~dB}$. Approximately a $28-\mathrm{dB}$ enhancement is achieved by the velocity stack.

The above analysis is for the ideal case in which we know exact multiple and primary velocities. For this ideal case, there is neither random nor other coherent noise, and each channel has an identical waveform. These ideal conditions are never met with real data.

\section{NOISE MODEL}

In order to evaluate the performance of the velocity stack in a "real" data situation, noise in the form of random static shifts, in the range of $4 \mathrm{~ms}$, and random amplitude with
Gaussian random noise were introduced into the CMP gather. The static shift was chosen for our noise model for the following reasons:

1. The velocity stack or other multiple-suppression technique keys on the alignment of signal and coherent noise, so the performance depends on the degree of misalignment.

2. Other kinds of misalignment, such as waveform changes due to different ghosting, can be modeled approximately by a static shift.

3. The effect of inaccurate multiple velocities can be treated as a static problem.

4. Static shifts account for a variation of streamer depth during data acquisition.

The performance of velocity stack with static shifts is also shown in figure 6 . The "b" parts of figure 6 represent the results of straight stacking, and the " $\mathrm{d}$ " parts of figure 6 represent the results of multiple suppression by the inverse velocity stack. Notice that static shifts on the order of $4 \mathrm{~ms}$ does not significantly affect the performance of straight stacking (compare the "a" parts with the "b" parts of fig. 6). However, the performance of the inverse velocity stack is highly dependent on the static shift. In the "d" parts of figure 6 , the primaries are still recognizable for model 1 , but not for model 3. The performance of the velocity stack under the influence of static shifts is shown in figure $7 B$. The strength of primary events in figure $7 A$ (noise free) and figure $7 B$ (static noise) are similar, but the amount of multiple suppression under the static shifts is on the order of 20 $\mathrm{dB}$ (it is about $40 \mathrm{~dB}$ in the noise-free case). In other words, if the multiple energy contaminated by static shifts is more than approximately $20 \mathrm{~dB}$ higher than that of primaries, it is difficult to enhance primaries over multiples using the inverse velocity stack.

Notice that the strength of multiple energy is almost the same as that of the primaries in figure 6, model 3, "d" part, after multiple suppression. Recalling that the multiple strength before the multiple suppression for model 3 is $20 \mathrm{~dB}$ higher than the primary, the signal-to-noise ratio for model 3 can be considered as a threshold for recognizing the primaries. However, in order to differentiate primaries from multiples, the amplitudes of primary events after multiple suppression should be a couple of decibels higher than those of the multiples. Figure 6, model 1, "d" part, whose multiple strength is about $14 \mathrm{~dB}$ higher than the primaries, clearly shows the primaries after multiple suppression even though there are 4-ms static shifts. Therefore, in processing real data sets, we could not enhance the primaries if the strength of multiples is about $17 \mathrm{~dB}$ greater than that of the primaries (see fig. 6, model 1, "d" part and fig. 6, model 3, "d" part).

Approximately a $20-\mathrm{dB}$ loss of performance of the velocity stack for the noise model is due to small static shifts. In theory, static shifts can be computed and static corrections can be applied before the velocity stack. One of the results of applying static shifts computed by non-surface-consistent 


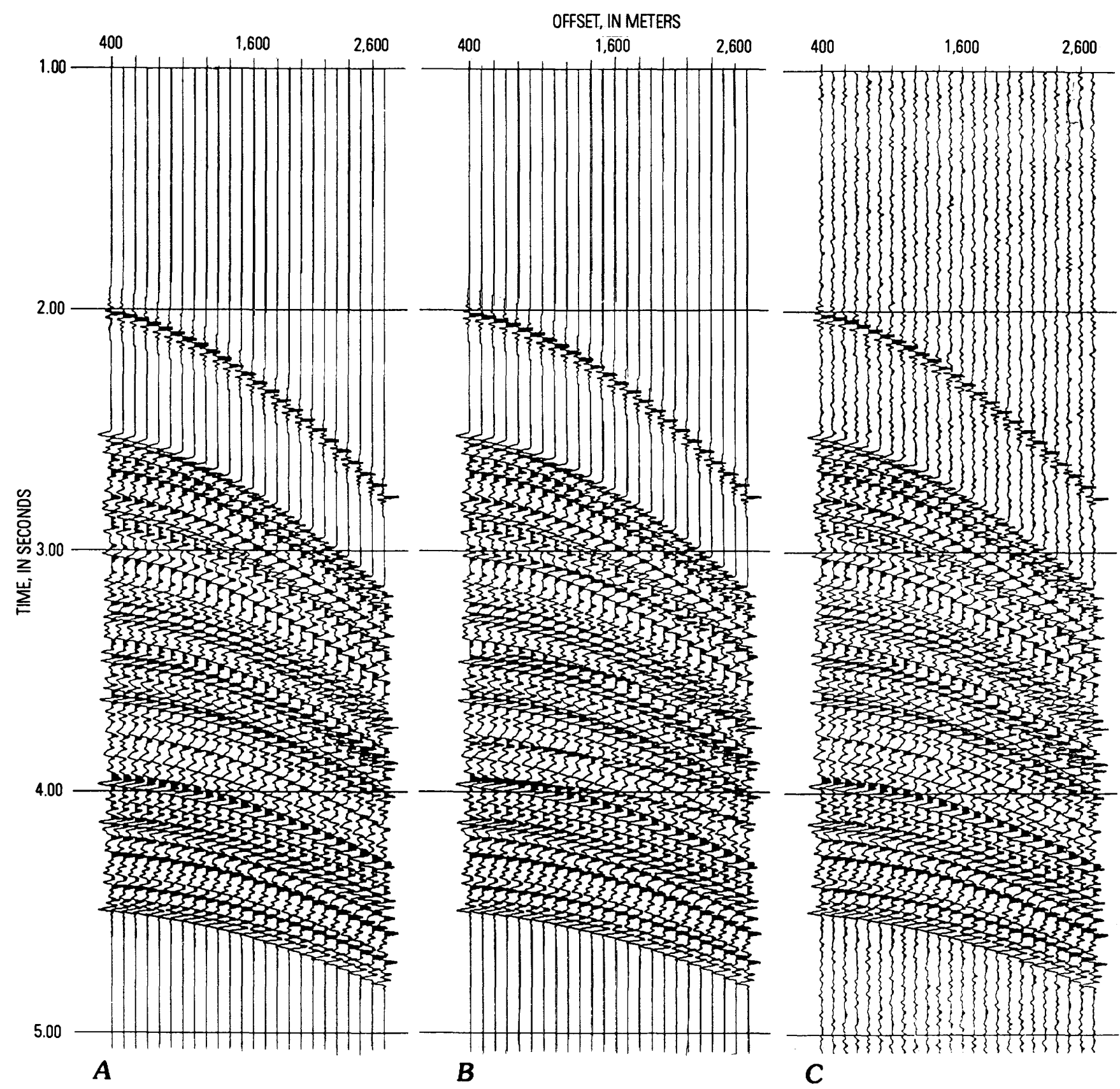

Figure 5. Models for multiple suppression. $A$, Multiple model 1: The multiple strength is about $14 \mathrm{~dB}$ higher than primaries; $B$, Multiple model 2: The multiple strength is almost the same as primaries; $C$, Additive random noise to model 1 . The ratio of primary signal to random noise (not coherent multiple noise) is about 1 .

static, or "trim static" in industry terminology, is shown in figure 8 for model 3. As expected, the result (fig. $8 D$ ) is comparable to the result for the noise-free model (fig. $8 B$ ).

The effect of random-amplitude noise in multiple suppression is analyzed using model 1 by adding Gaussian random noise with maximum amplitudes to those of primaries (fig. 5C). The result of multiple suppression using an inverse operator is shown in figure 9 . Figure $9 A$ shows the result of straight stacking with random noise, and figure $9 B$ shows the result of straight stacking with both random noise and static shifts in the range of $4 \mathrm{~ms}$. There is not much difference between figures $9 A$ and $9 B$. Both are too noisy to recognize primary events. The result of applying an inverse operator to the random noise section without static shifts is shown in figure $9 C$. All primary events are clearly observable. The effect of both random-amplitude 

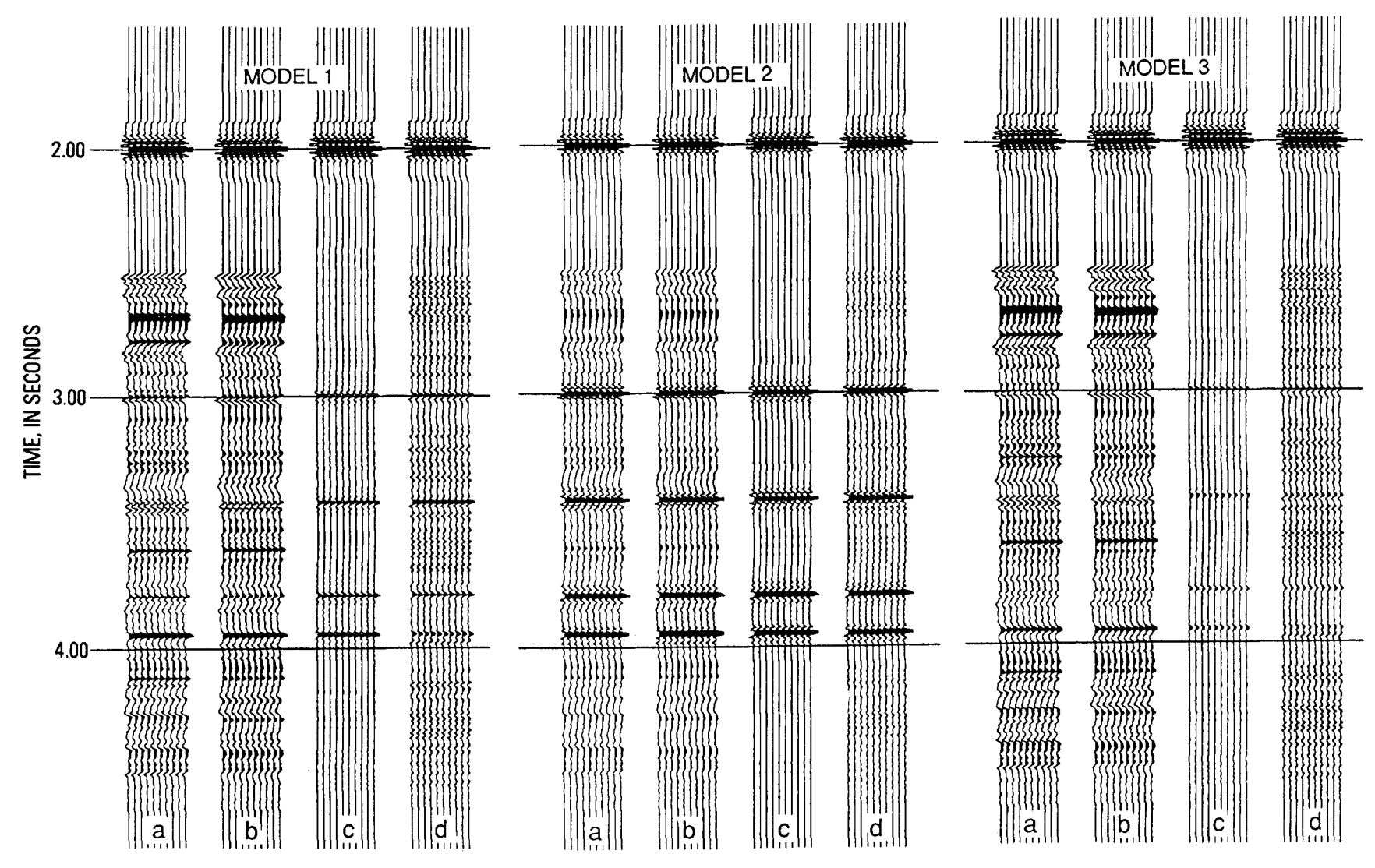

Figure 6. Multiple suppression for three multiple models with and without static shifts in the range of $4 \mathrm{~ms}$ using an inverse operator in the velocity stack: a, Straight stack without static shifts. b, Straight stack with static shifts. c, Multiple suppression without static shift. d, Multiple suppression with static shifts.

noise and static shifts is shown in figure $9 D$, and the result of application of static correction is shown in figure $9 E$. Comparing with figure 6 for model 1 , it is obvious that noise in the form of 4-ms static shifts has a more pronounced effect than an equal amount of random noise.

The result of multiple suppression using the transpose operator is shown in figure 10 . In comparing figures $9 C$ and $9 E$ with figures $10 C$ and $10 E$, the advantage of the inverse operator over the transpose operator is evident. But when there is too much noise, the results shown in figure $9 D$ and figure $10 D$ imply that there is not much difference between the inverse and transpose operators. Hence, for high-quality input data, the inverse operator works better than the transpose operator. But, as the amount of noise (particularly in the form of static time shifts) increases, the advantage of the inverse operator over the transpose operator decreases.

\section{REAL DATA EXAMPLE}

Figure 11 shows an original stacked section generated using a standard processing sequence. This data set was acquired in Lake Baikal using a tuned air-gun array as a source. Unstacked data consists of 24-fold CMP gathers with a near offset distance of $400 \mathrm{~m}$ and a far offset distance of $2,775 \mathrm{~m}$.

Our model study indicates that correcting for static shifts is a very important step in suppressing multiple energy. Thus, one additional step was added to the steps outlined at the outset of the multiple suppression section: static computation and application after step 1. The static computation was performed in the CMP domain.

Figure 12 shows the result of multiple suppression. Figure $12 A$ is the result of applying two-dimensional velocity filtering, or F-K filtering, a technique similar to the one described by Ryu (1982), and figure $12 B$ shows the result of the application of the inverse velocity stack. For the inverse velocity stack, the multiples are modeled with seven slownesses, and the range of parabolic moveouts is $-20 \mathrm{~ms}$ to 40 $\mathrm{ms}$ at the far offset. Both techniques performed adequately for the multiple suppression below about $4,600 \mathrm{~ms}$. Overall signal-to-noise ratio, however, is better in the section produced by the application of inverse velocity stack. The details of figure 12 near CMP 2,500 is shown in figure 13, and this figure clearly demonstrates that the inverse velocity 


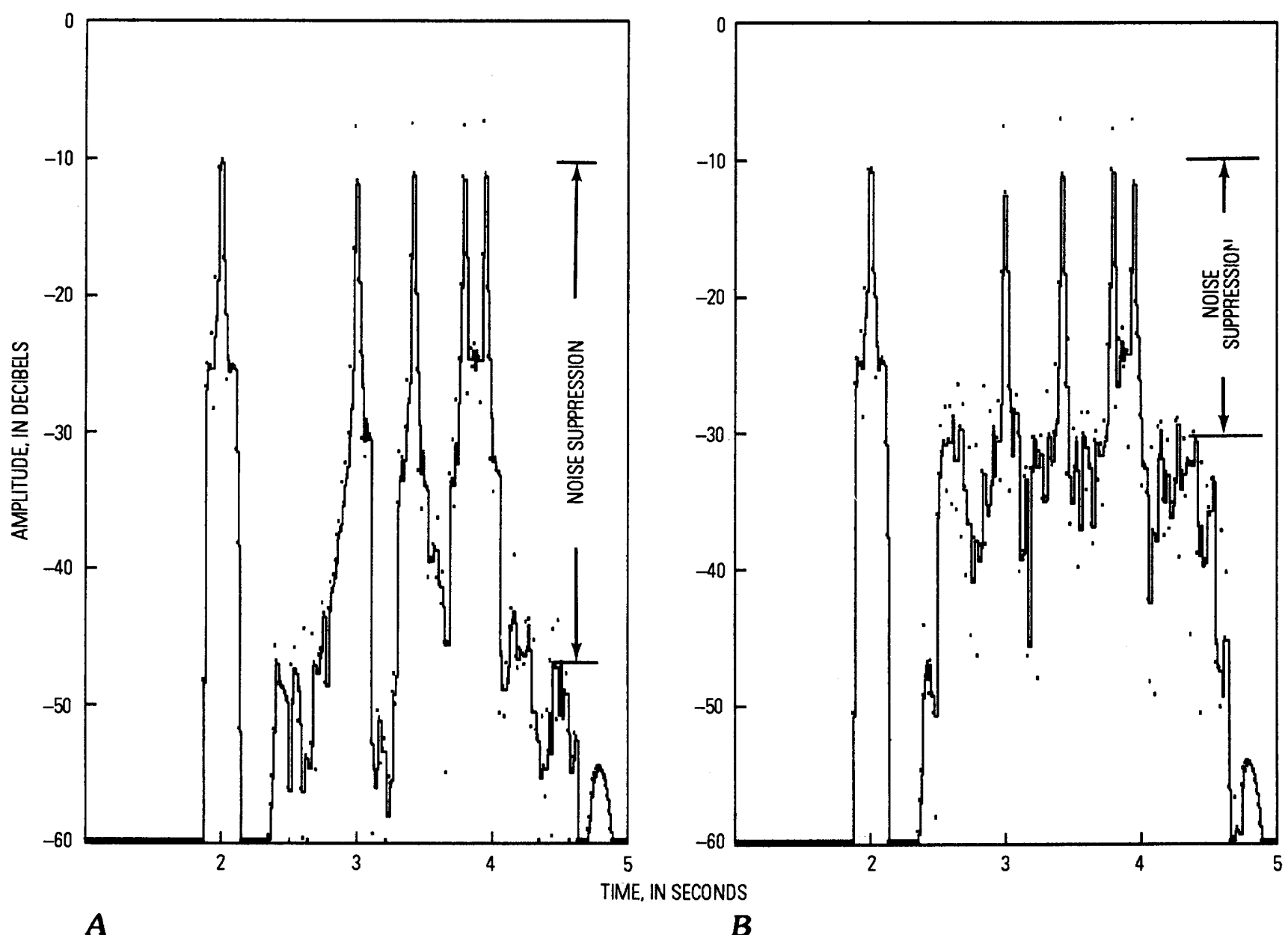

Figure 7. Amplitude analysis of multiple suppression for model 2 using an inverse operator in the velocity stack. Each dot represents a root-mean-square (RMS) amplitude within $16 \mathrm{~ms}$, and solid lines are running medians of three amplitudes. $A$, Without static shift; $B$, With static shift in the range of $4 \mathrm{~ms}$.

stack outperforms the F-K operation in multiple suppression. Notice much stronger and clearer dipping events are imaged in figure $13 B$.

We tested many seismic profiles using the inverse velocity stack versus the F-K domain approach. In almost every case, the inverse velocity stack worked better than the F-K domain approach, particularly for data sets with high noise and low frequency. A similar conclusion was reached by Geist and others (1990) in their study of longperiod ( $>1 \mathrm{~s}$ ), first-order, multiple removal over a continental slope. Better performance of the inverse velocity stack for the lower frequency range has an important implication for deep crustal studies. As mentioned in Hampson (1986), the better performance of the inverse velocity stack is due to the fact that inverse operators work well for all offsets, whereas the F-K approach leaves significant multiple energy on the near-offset traces.

\section{COHERENCY FILTERING}

It is well known that stacking suppresses random noise proportional to the root of the number of traces in the stack. If all reflections are horizontal, this is a good method for enhancing signal-to-noise ratio (this is the fundamental concept applied in CMP stacking). In the stacked section, this is not an appropriate method because there are numerous nonhorizontal events. In order to enhance dipping events, stacking should be done along different moveouts. Stacking along the linear moveouts can be effectively implemented using a slant stack in the $\tau-p$ domain. This kind of coherency filtering is necessary for data with a low signal-to-noise ratio.

In this section we will discuss coherency filtering in the context of model extraction from noisy data. As indicated in equation 6 , the model space can be estimated by applying the inverse operator to the offset-domain data set. This estimated 


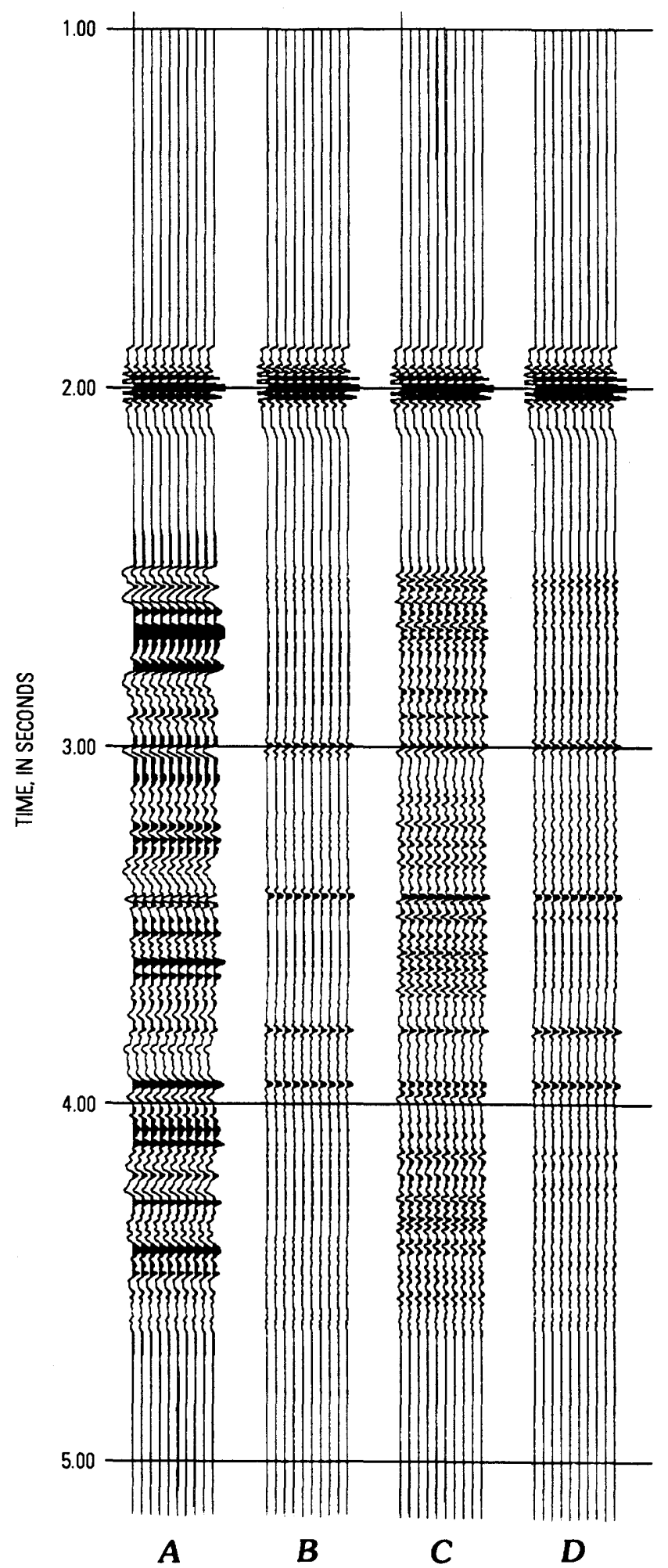

Figure 8. Multiple suppression for model 3 using an inverse operator in the velocity stack. $A$, Straight stack without static shift; $B$, Multiple suppression without static shift; $C$, Multiple suppression with static shift in the range of $4 \mathrm{~ms} ; D$, Multiple suppression with static shift after correction with "trim static" (see text) in the CMP domain.

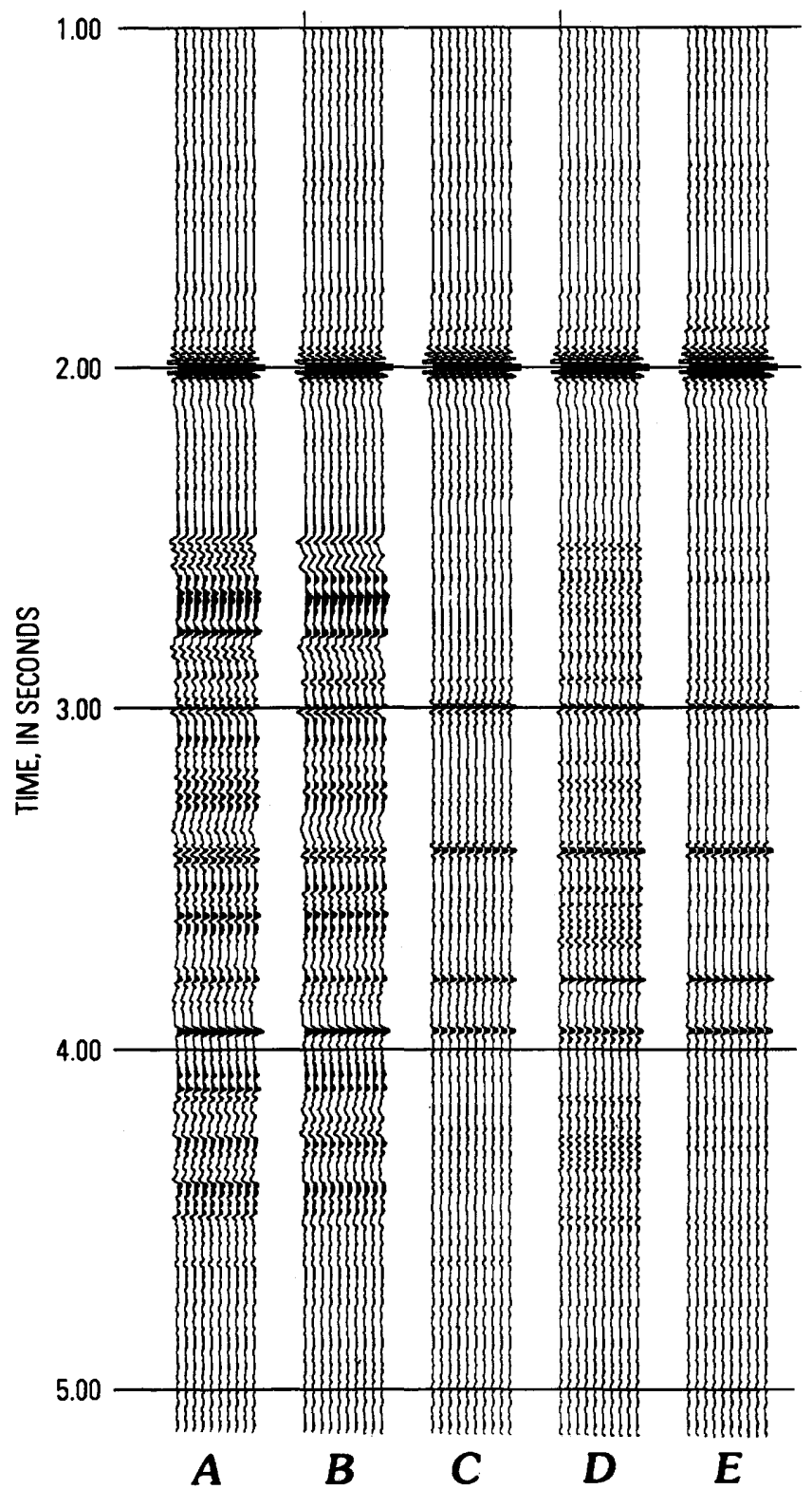

Figure 9. Multiple suppression for model 1 with randomamplitude noise and static shifts using an inverse operator in the velocity stack. Primary signal-to-random-noise ratio is about 1 , and the range of static shifts is $\pm 4 \mathrm{~ms}$. $A$, Straight stack with random noise only; $B$, Straight stack with random noise and static shift; $C$, Multiple suppression with random noise only; $D$, Multiple suppression with random noise and static shift; $E$, Multiple suppression with random noise and static shift after static correction with "trim static" (see text) in the CMP domain. 


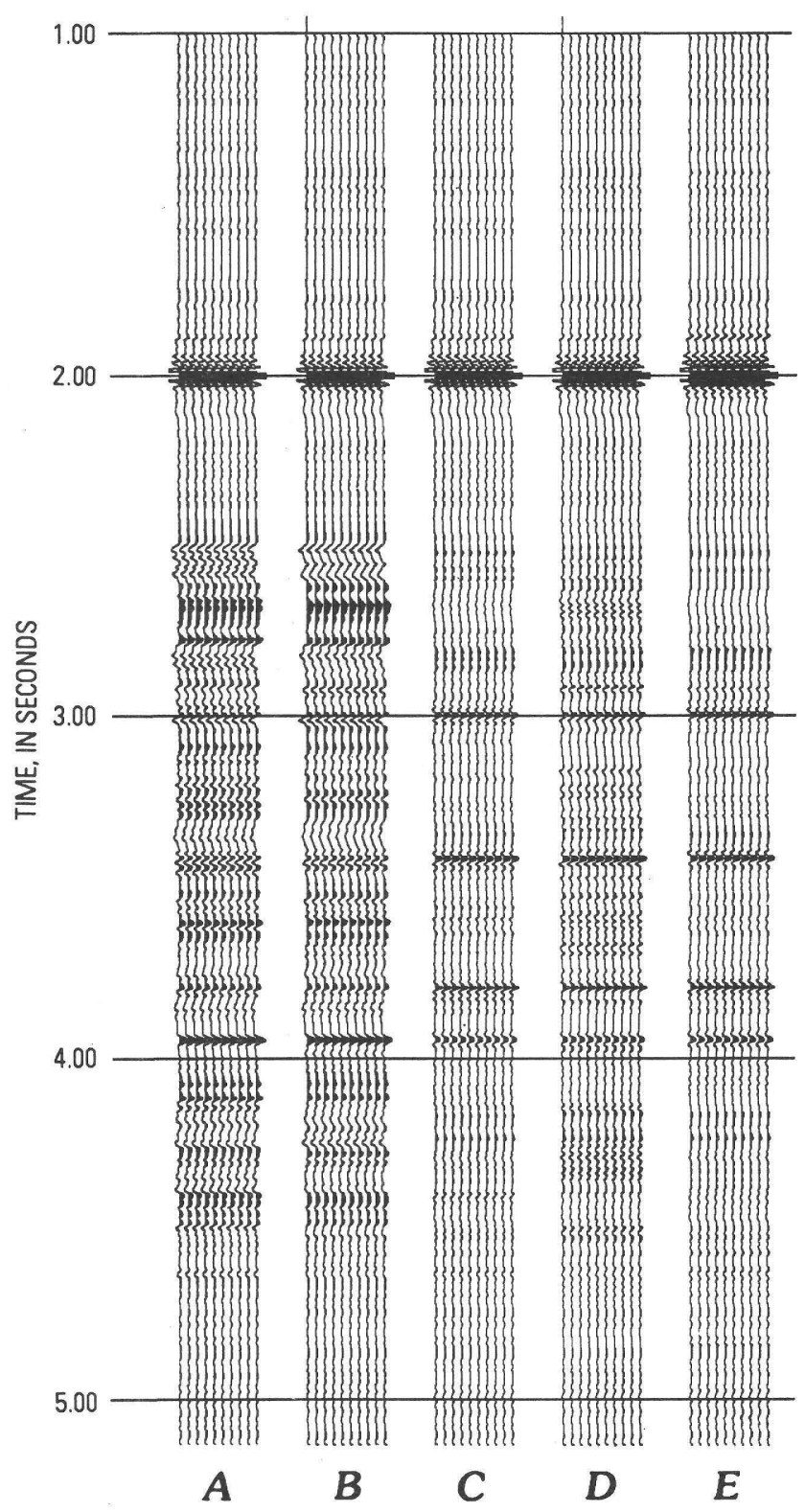

Figure 10. Multiple suppression for model 1 with randomamplitude noise and static shifts using a transpose operator in the velocity stack. Primary signal-to-random-noise ratio is about 1 , and the range of static shifts is $\pm 4 \mathrm{~ms}$. $A$, Straight stack with random noise only; $B$, Straight stack with random noise and static shift; $C$, Multiple suppression with random noise only; $D$, Multiple suppression with random noise and static shift; $E$, Multiple suppression with random noise and static shift after static correction with "trim static" (see text) in the CMP domain.

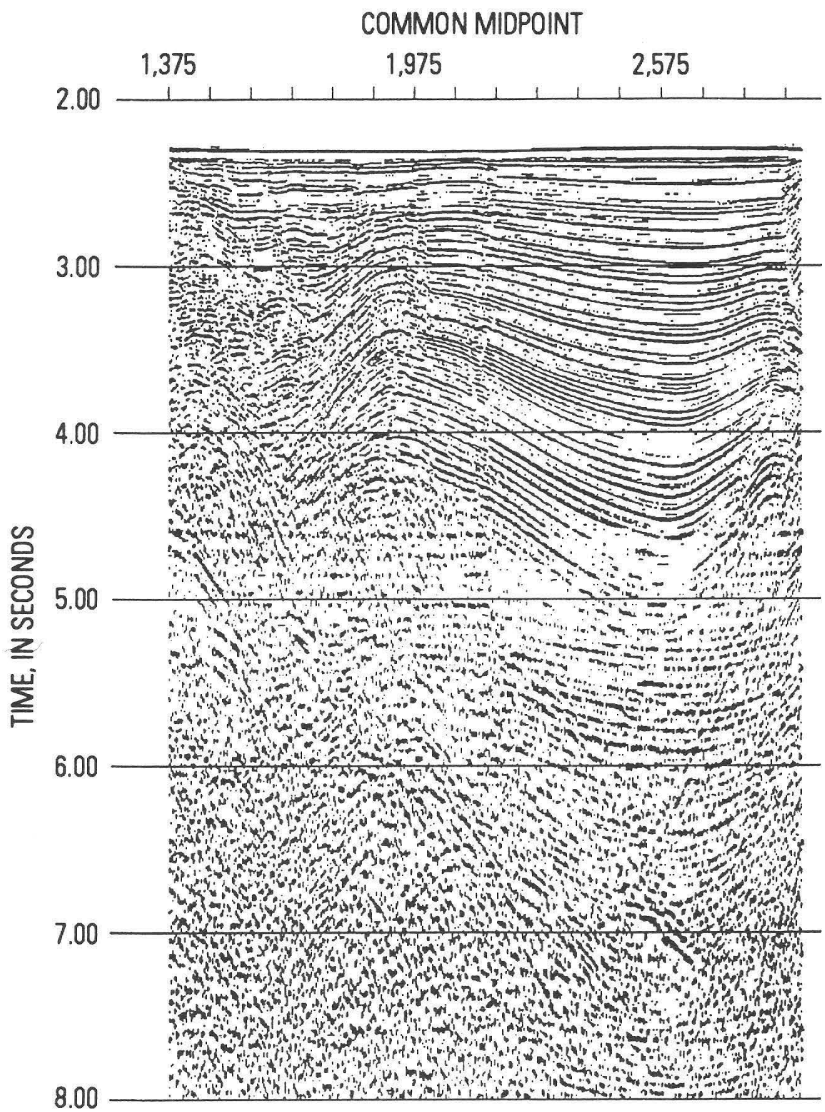

Figure 11. Straight stacked section of a profile acquired at Lake Baikal in the fall of 1992 . Notice strong, first-order, water-bottom multiple and pegleg multiples after $4,600 \mathrm{~ms}$. See text for acquisition parameters. 


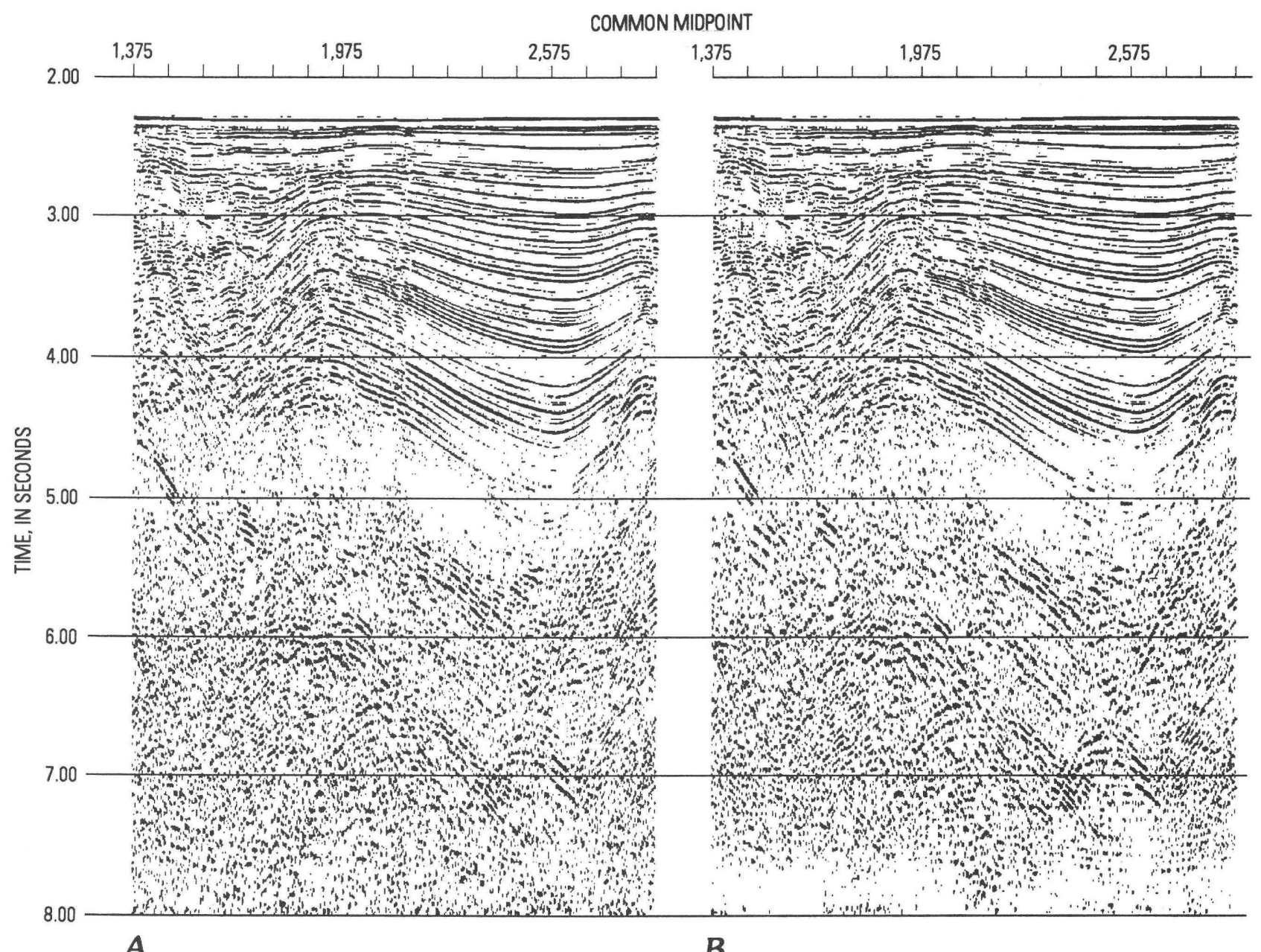

Figure 12. Multiple suppression after applying static correction to data shown in figure 11. A, Multiple suppression using twodimensional frequency-wavenumber domain filtering; $B$, Multiple suppression using an inverse operator in the velocity stack.

model or signal can be inverse-transformed back to the offset domain data set using equation 4 without the noise term. The presence of the noise in equation 4 indicates that the transform generated by equation 6 is not a complete space. Some of the noise, $n(h, t)$, that has not been included in $\mathbf{U}$ is lost when transforming back to the offset domain. This operation can be written as:

$$
\mathbf{D} \stackrel{G_{i j}}{-} \mathbf{U}-\hat{\mathbf{D}}
$$

where

$\hat{\mathbf{D}}$ is the coherency-filtered output.

Figure $14 \mathrm{~A}$ shows the result of applying an inverse operator as a coherency filter to the section shown figure $12 B$, and figure $14 B$ shows the result of applying a transpose operator. The inverse or transpose operator was designed using linear moveout with 11 slowness steps and five offsets. The range of linear moveout at the far offset is $\pm 24 \mathrm{~ms}$ (the offset of the center trace is $0 \mathrm{~m}$ ). The output is estimated at the center trace. Both figures $14 A$ and $14 B$ show a reduction of background random noise or the enhancement of local coherency estimated within five traces. Details of coherency filtering in the time domain are shown in figure 15, and figure 16 shows the frequency-wavenumber domain representation of the data shown in figure 15. The low-frequency enhancement and mixing effects produced by the transpose operator are apparent in figures 15 and 16.

\section{TRACE INTERPOLATION}

The concept of coherency filtering by inverse velocity stack can be applied to trace interpolation. The only difference is the application of the $\mathbf{L}$ operator in equation 11 . In equation 11, the element of $\mathbf{L}$ applying to model space $\mathbf{U}$ to transform back to the offset domain is the same element used 


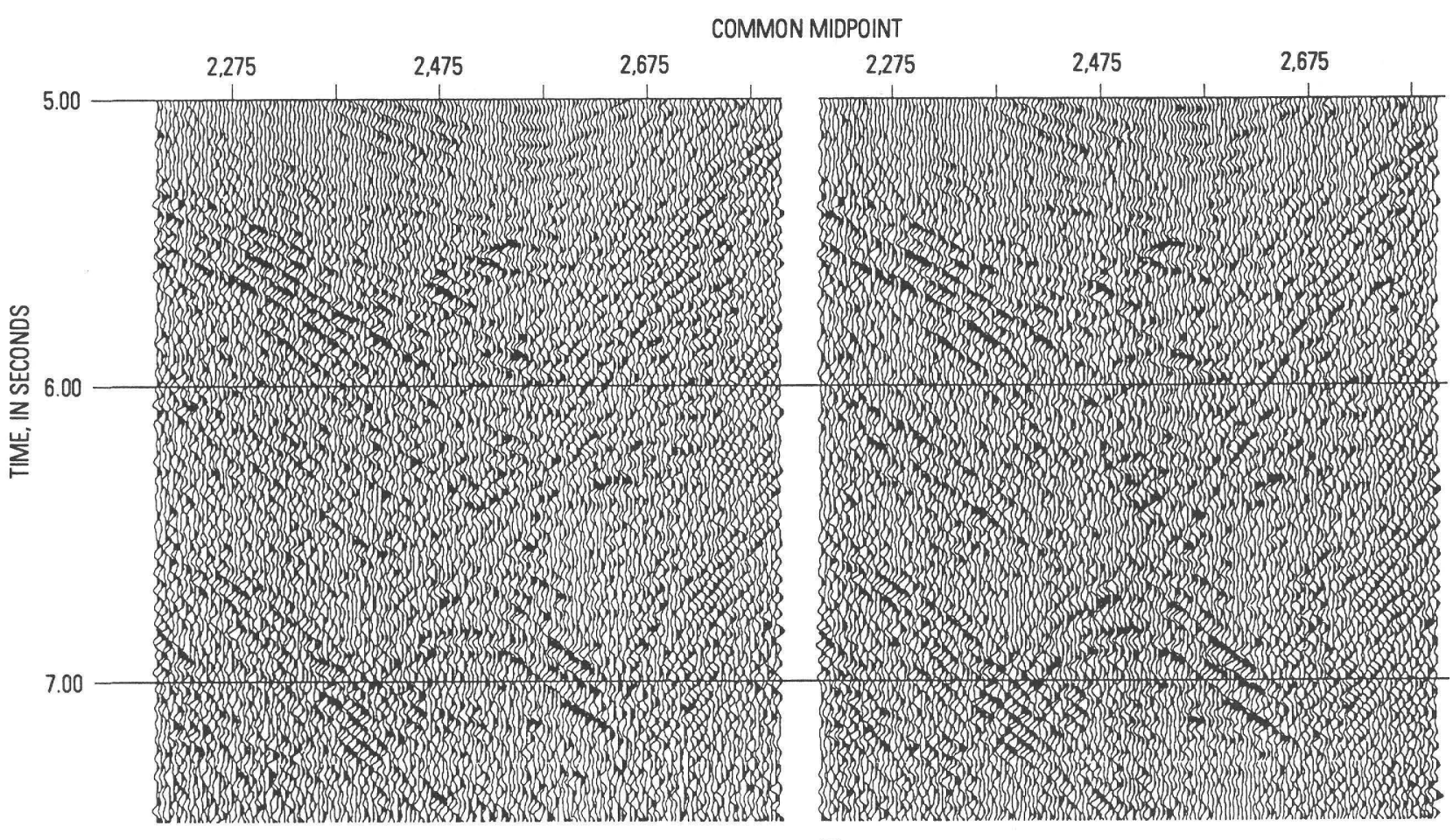

A

B

Figure 13. Expanded plot of figure 12 near CMP 2,500. A, Multiple suppression using two-dimensional frequency-wavenumber domain filtering; $B$, Multiple suppression using an inverse operator in the velocity stack.

for computing $\mathbf{G}$. But in trace interpolation, a different $\mathbf{L}$ can be used for the output. In other words, a different offset value, not included in the input data, can be used for the interpolation. Assume that a trace is interpolated using two input traces, and the interpolation is done at the middle of two traces using linear moveout. Then the $\mathbf{L}$ used for computing $\mathbf{G}$ can be written as:

$$
\mathbf{L}=\left(\begin{array}{l}
e^{-i \omega s_{1} h_{1}} e^{-i \omega s_{2} h_{1}} \\
e^{-i \omega s_{1} h_{2}} e^{-i \omega s_{2} h_{2}}
\end{array}\right)
$$

The element $\mathbf{L}^{*}$ for the interpolation can be written as:

$$
\mathbf{L}^{*}=\left(e^{-i \omega s_{1} h_{1}^{*}} e^{-i \omega s_{2} h_{1}^{*}}\right)
$$

where

$$
h^{*}=\left(h_{1}+h_{2}\right) / 2
$$

An example of trace interpolation using an inverse operator is given in figure 17. The trace interval of figure 17 is $25 \mathrm{~m}$, and five traces were used for the interpolation. An inverse operator was designed to pass seismic events whose linear moveouts are within \pm 4 ms per trace. Highly dipping events at the left side and random noises are reduced in the interpolated section (fig. 17B). Also, trace mixing, which is obvious when using the transpose operator, is not apparent in the interpolated section.

\section{VELOCITY ANALYSIS}

Moveouts in the CMP domain depend on the interval velocities of the layered Earth, and the model space is estimated in the slowness domain. Thus, the inverse velocity stack is a logical choice for velocity analysis. When using hyperbolic moveouts, the slowness can be directly related to the Earth's velocity. But, in using parabolic moveouts, it only provides a pseudo or apparent velocity after NMO correction with velocity, $v_{c}$ (or slowness, $p_{c}$-see eq. 8).

Parameter "sh" in parabolic moveouts is presented as the moveout time at the far-offset distance; thus, a relationship is required to derive an actual velocity from the velocity stack using parabolic moveouts. The relationship between apparent and true velocity can be derived from equation 8 :

$$
\frac{1}{v^{2}}=\frac{1}{v_{r}^{2}}+\frac{1}{v_{c}^{2}}
$$

Using the parameter $s$ :

$$
v^{2}=\frac{v_{c}^{2}}{2 \tau s v_{c}^{2}+1}
$$

Because the parameter is given by the moveout time at the far offset, equation 14 can be written as: 


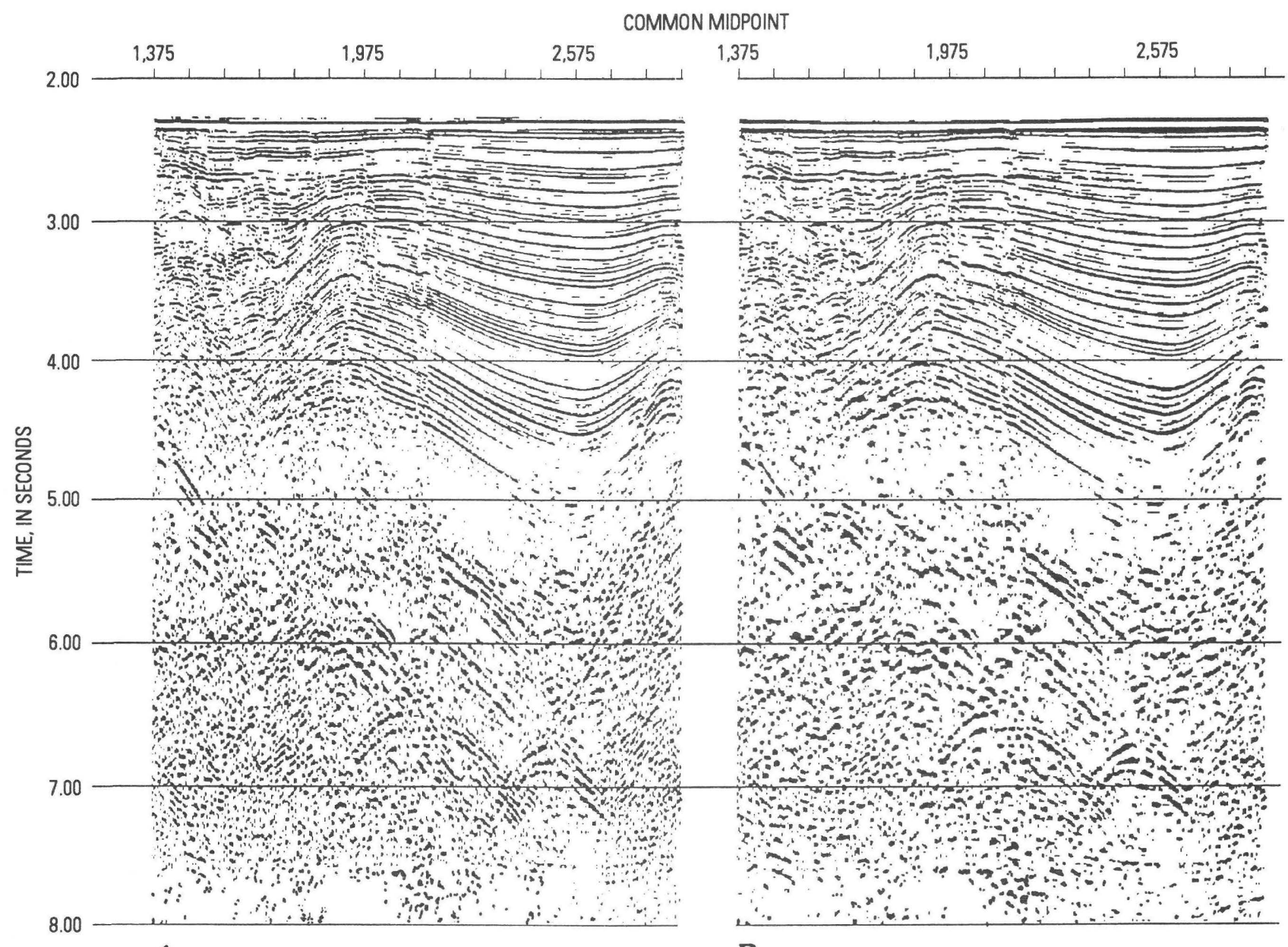

A

B

Figure 14. Example of coherency filtering of the section shown in figure $12 B$. Coherency filter was designed using five offsets with the moveout time at the far offset of $\pm 24 \mathrm{~ms}$. The offset at the center trace is $0 \mathrm{~m}$, and the trace interval is $50 \mathrm{~m}$. $A$, Inverse operator; $B$, Transpose operator.

$$
v=\sqrt{\frac{v_{c}^{2}}{ \pm M_{t} 2 \tau v_{c}^{2} / h_{\max }^{2}+1}}
$$

where

$M_{t}$ is moveout time at the far offset, and

$h_{\max }$ is the far-offset distance.

As indicated in equation 15 , the true velocity depends on the zero-offset time as well as the correction velocity for NMO and moveout time.

A velocity analysis for the noise-free model shown in figure $5 B$ is given in figure 18 with linearly increasing velocity of $v_{c}=1,500 \mathrm{~m} / \mathrm{s}$ at $2,500 \mathrm{~ms}$ and $v_{c}=1,700 \mathrm{~m} / \mathrm{s}$ at 4,500 $\mathrm{ms}$. The range of moveout times at the far offset is $-240 \mathrm{~ms}$ to $120 \mathrm{~ms}$, with a $12-\mathrm{ms}$ interval. The output of the application of the inverse operator, shown in figure $18 \mathrm{~A}$, clearly shows linearly increasing velocity for the multiples and four primary events. The moveout time of $\mathrm{P} 4$ is $-168 \mathrm{~ms}$ at twoway time of $3,950 \mathrm{~ms}$. Putting this number into equation 15 , the correct velocity by a parabolic moveout is $2,197 \mathrm{~m} / \mathrm{s}$, which is very close to the input velocity of $2,200 \mathrm{~m} / \mathrm{s}$. However, the output from the transpose operator or conventional velocity analysis, shown in figure $18 B$, show a smearing effect of the adjacent moveouts. The above analysis was applied to the noisy model, random noise (primary signal-torandom-noise ratio of about 2), and static shifts (in the range of $4 \mathrm{~ms}$ ), and the result is shown in figure 19. Although the result from the inverse operator is better than the result from the transpose operator, the difference is not as dramatic as that shown in figure 18 .

An application to a real data set is shown in figure 20. The result using the application of an inverse operator (fig. $20 A$ ) produces better resolution but is noisier than the result produced by the transpose operator (fig. 20B) 


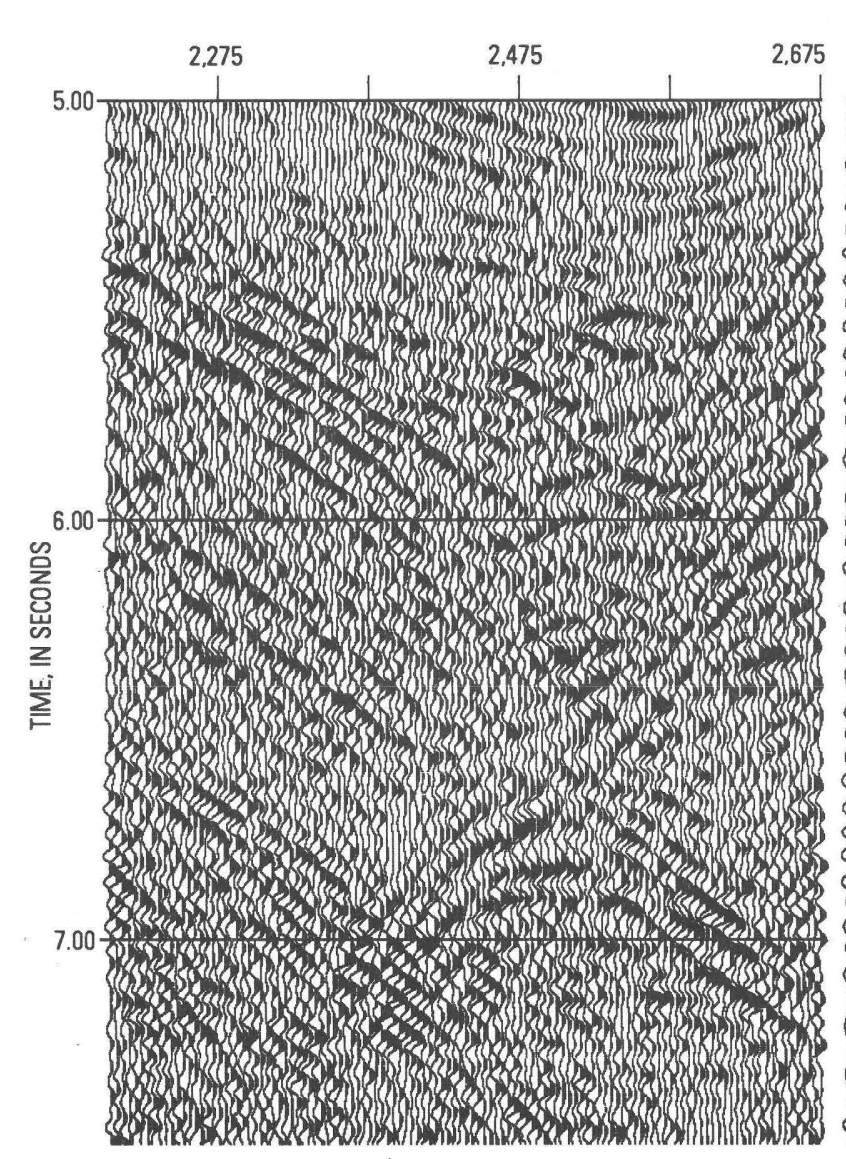

A

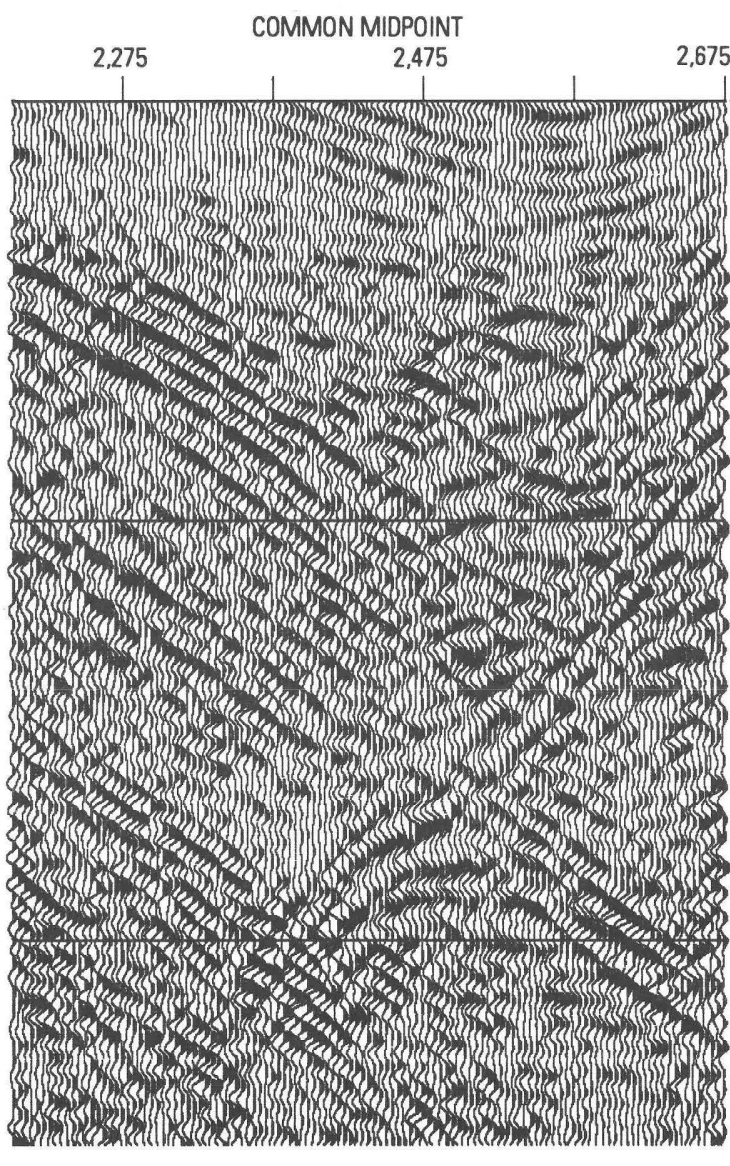

B

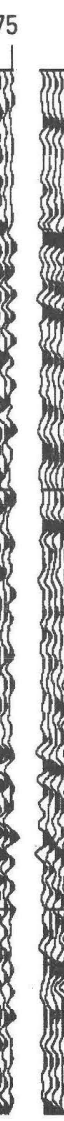

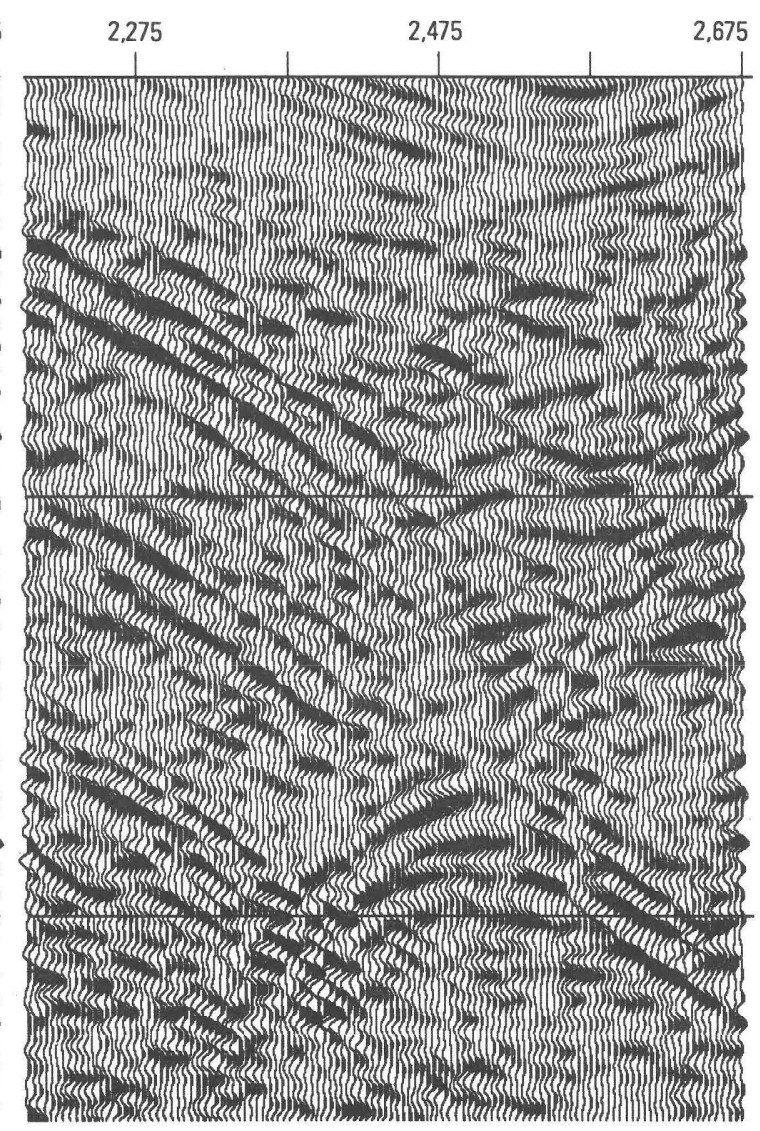

C

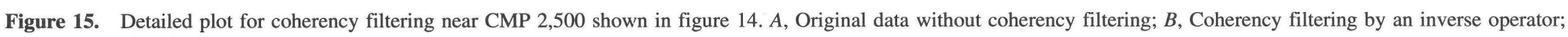
$C$, Coherency filtering by a transpose operator. 


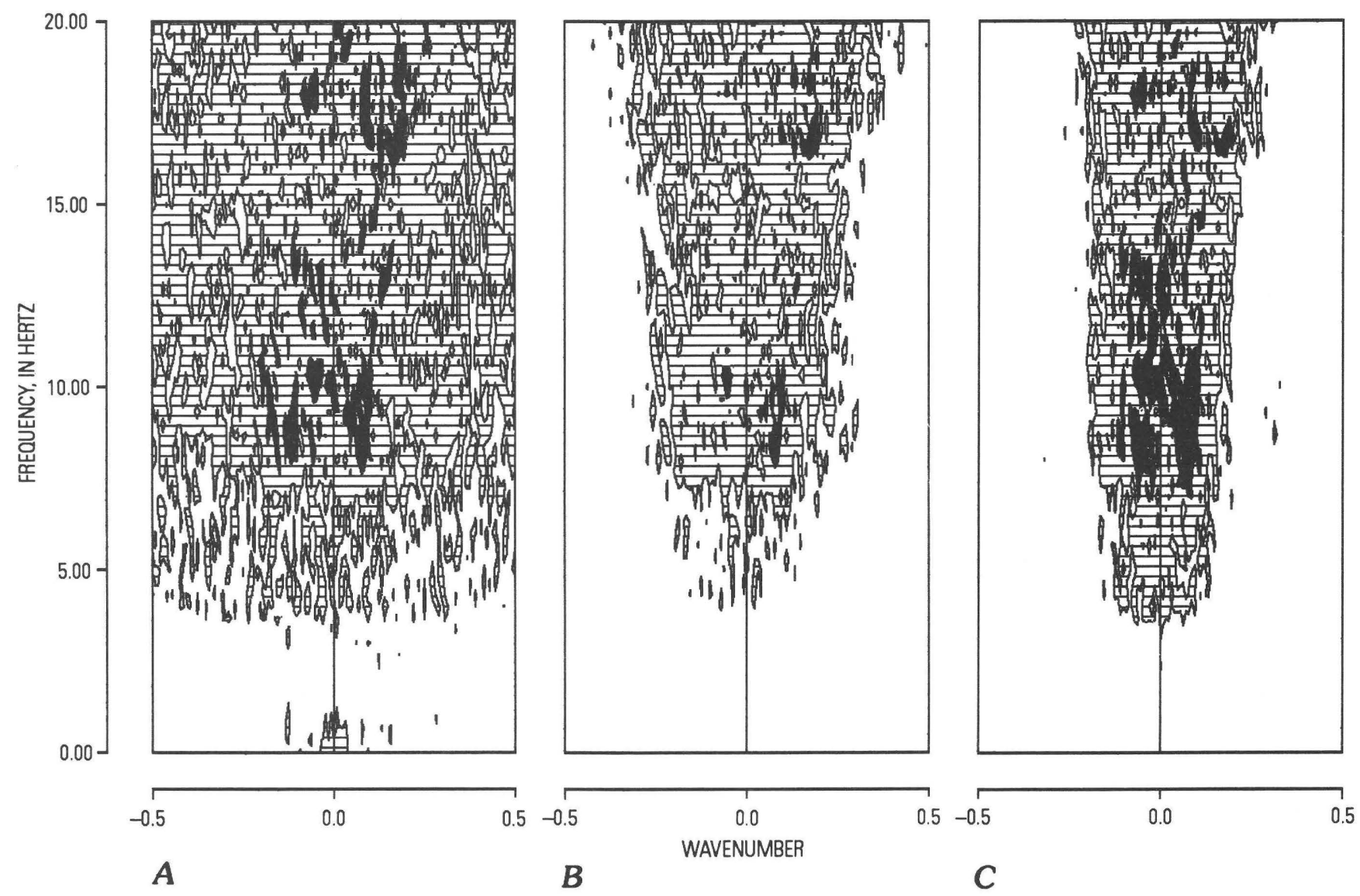

Figure 16. Two-dimensional frequency-wavenumber analysis for the data shown in figure 15. A, Original data without coherency filtering; $B$, Coherency filtering by an inverse operator; $C$, Coherency filtering by a transpose operator.

\section{DISCUSSION}

Many conventional seismic processing methods are based on the transpose operator or conjugate operator (Claerbout, 1992). Although the transpose operator applied to perfect data generally gives an imperfect result, sometimes the transpose operator performs better than the inverse operator because the conjugate operator tolerates imperfections of input data owing to the limited aperture, truncation, nonstationary noise, missing data, etc. On the other hand, an inverse operator gives a perfect result for a perfect data set but has the possibility of noise amplification when using actual data and requires more information to do a better job. As can be seen from equation 6, the transpose operator is the first step of inversion, and, if $\left(\mathbf{L}^{T} \mathbf{L}\right)^{-1}$ is close to an identity matrix, the inverse operator can be approximated by a transpose operator.

To reduce noise amplification of the inverse operator applied to an imperfect data set or to stabilize the inverse operator, a random noise factor, $\Lambda$, such as the one used for spiking deconvolution, can be applied. When implementing a computer program for equation $6,\left(\mathbf{L}^{T} \mathbf{L}+\Lambda\right)^{-1}$ is used for the computation of the inverse operator. All examples in this paper are generated by adding 1 percent of each diagonal element to the diagonal terms. The amount of noise can be adjusted to tune the performance of the inverse operator. Thorson and Claerbout (1985) defined this form of inverse as "stochastic inverse" (Aki and Richards, 1980) and suggest using the ratio of noise variance to variance of the points in the model space for $\Lambda$. The method proposed by Thorson and Claerbout (1985) may provide an optimum inverse operator for each data set, but the operator cannot be pre-computed because $\Lambda$ cannot be determined without data.

Two basic assumptions in inverse velocity stacking are that, along various moveouts, events are aligned and have uniform amplitudes. Multiple suppression using real data sets shows improved results after static time corrections are applied to improve alignment. However, the improvements were marginal in most cases. One example is shown in figure 21 . This suggests that simple static corrections alone will not suffice in certain cases. This is because our waveform changes in a CMP gather. This waveform change violates the 


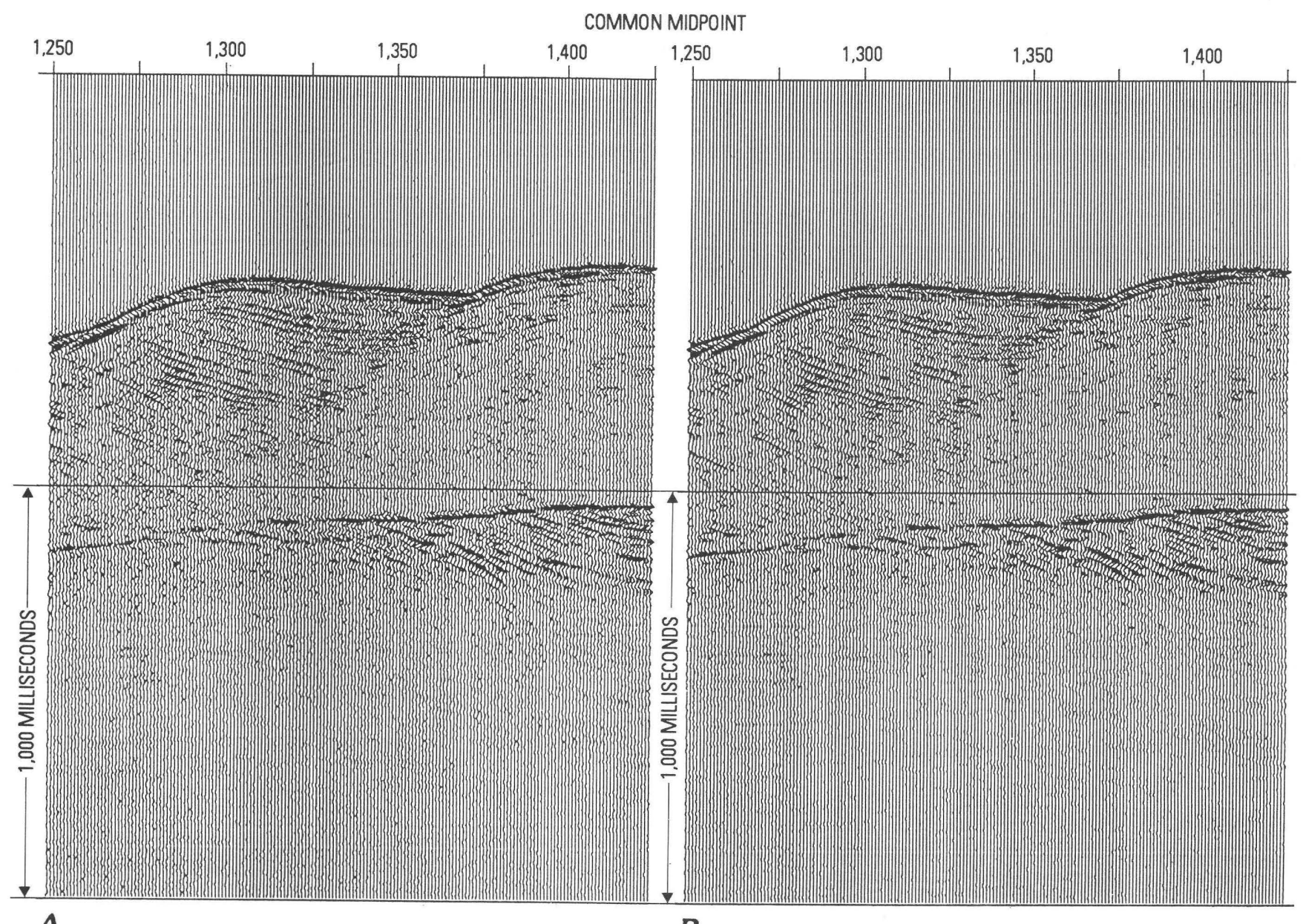

A

B

Figure 17. Example of trace interpolation using an inverse operator. Trace interval is $25 \mathrm{~m}$, and five traces are used for the interpolation. $A$, Input data; $B$, Interpolated data.

basic assumptions of inverse velocity stacking. Thus, preprocessing, such as amplitude balancing and waveform deconvolution, will be beneficial to the performance of the inverse operator.

When inverse velocity stacking is applied for irregular offset gathers similar to land data or in a marine data set, whose CMP fold is less than the number of channels, it is impractical to compute different inverse operators for each offset gather. For example, 24-fold marine data acquired with 96 channels have 4 sets of offsets in the CMP domain, and 4 sets of inverse operators are required. To handle irregular geometries, one effective method is to apply differential NMO correction to a reference offset gather.

The estimation of model space from the noisy input data by means of the velocity stack is defined as a process of coherency filtering. Coherency filtering improves the signalto-noise ratio, as shown in figure 15 , and helps subsequent processing steps, such as migration. Most signal-enhancement techniques use weighted velocity stacks. Kong and others (1985) proposed a nonlinear signal estimator by multiplying semblance (Taner and Koehler, 1969) to the slantstacked data and inverse-transforming the weighted slantstack data. Monk (1992) presented a diversity slant stack, which is a weighted slant stack based on the power in the slant-stack domain. Both Kong and others (1985) and Monk (1992) applied a transpose operator in equation 11. In order to preserve the original frequency content, which is altered by applying a transpose operator, Monk (1992) and Kong and others (1985) applied an appropriate Rho filter (Claerbout, 1984) to the output. The main purpose of weighting, proposed by Monk (1992), is to reduce the smearing effect of the transpose operator and to modify the transpose operator, more or less, to look similar to an inverse operator. A weighted slant stack using an inverse operator provides an alternative to the conventional approach to signal enhancement. The price we have to pay for a weighted slant stack for coherency filtering in this formulation is that $\mathbf{U}$ should be converted in the $\tau-p$ domain to compute and apply weight. 


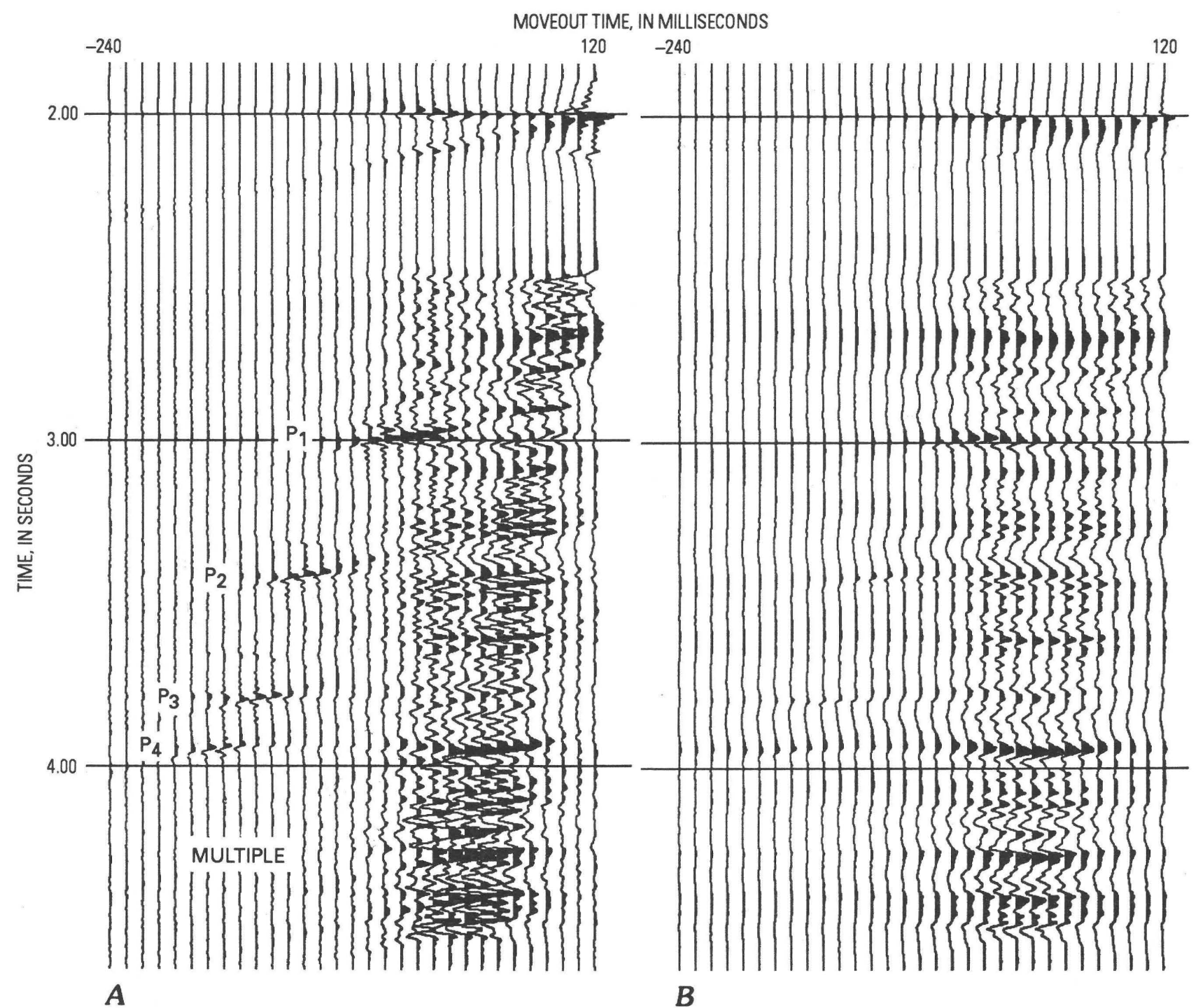

Figure 18. Example of velocity analysis using the noise-free model shown in figure $5 B$. The range of moveout time at the far offset is between $-240 \mathrm{~ms}$ and $120 \mathrm{~ms}$, with a $12-\mathrm{ms}$ interval. The correction velocities for NMO to approximate parabolic moveouts are linearly increasing velocities with $1,500 \mathrm{~m} / \mathrm{s}$ at $2,500 \mathrm{~ms}$ to $1,700 \mathrm{~m} / \mathrm{s}$ at $4,500 \mathrm{~ms}$. $A$, Inverse operator; $B$, Transpose operator.

The interpolation method presented here by means of the velocity stack implicitly uses a local coherency and dip estimation (or slowness in this paper) and is subject to the aliasing problem when interpolation distance is too large. The formulation of interpolation is cast as a prediction in the offset domain in this paper, but the essential operation is similar to interpolating the amplitude along the direction of lateral coherence of each event (Barden, 1987; Larner and others, 1981). This approach differs from Spitz (1991), who derived a method based on the fact that linear events may be interpolated exactly, regardless of spatial interval, without any information regarding their true dip. He interpolated traces using forward-backward, one-step prediction filters in the frequency domain. However, the proposed method has the ability to keep high-frequency components (as opposed to other interpolation methods based on the transpose operator).

One problem for the velocity analysis of real data using an inverse operator is low signal-to-noise ratio compared to the output by a transpose operator. One way to overcome this difficulty is to adjust the random noise component when deriving the inverse operator. More random noise in the design of the inverse operator increases the signal-to-noise ratio but also increases lateral smearing. An optimum tradeoff between resolution and smearing can be found by trial and error. Figure 22 shows a velocity analysis with an inverse operator using 2 percent noise for $\Lambda$, instead of 1 percent used for figure $20 \mathrm{~A}$ with static corrections. The output 


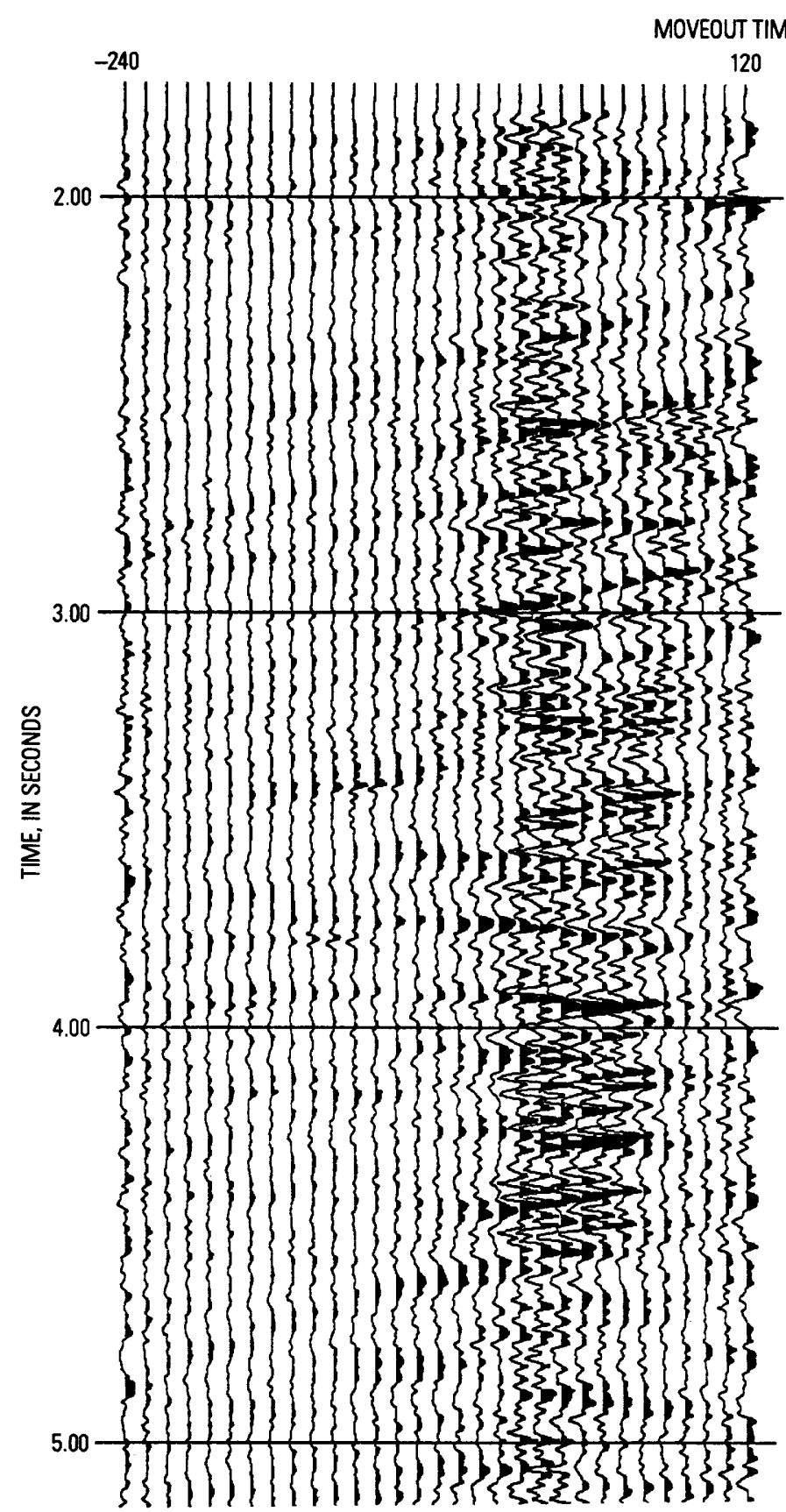

A
$-240$
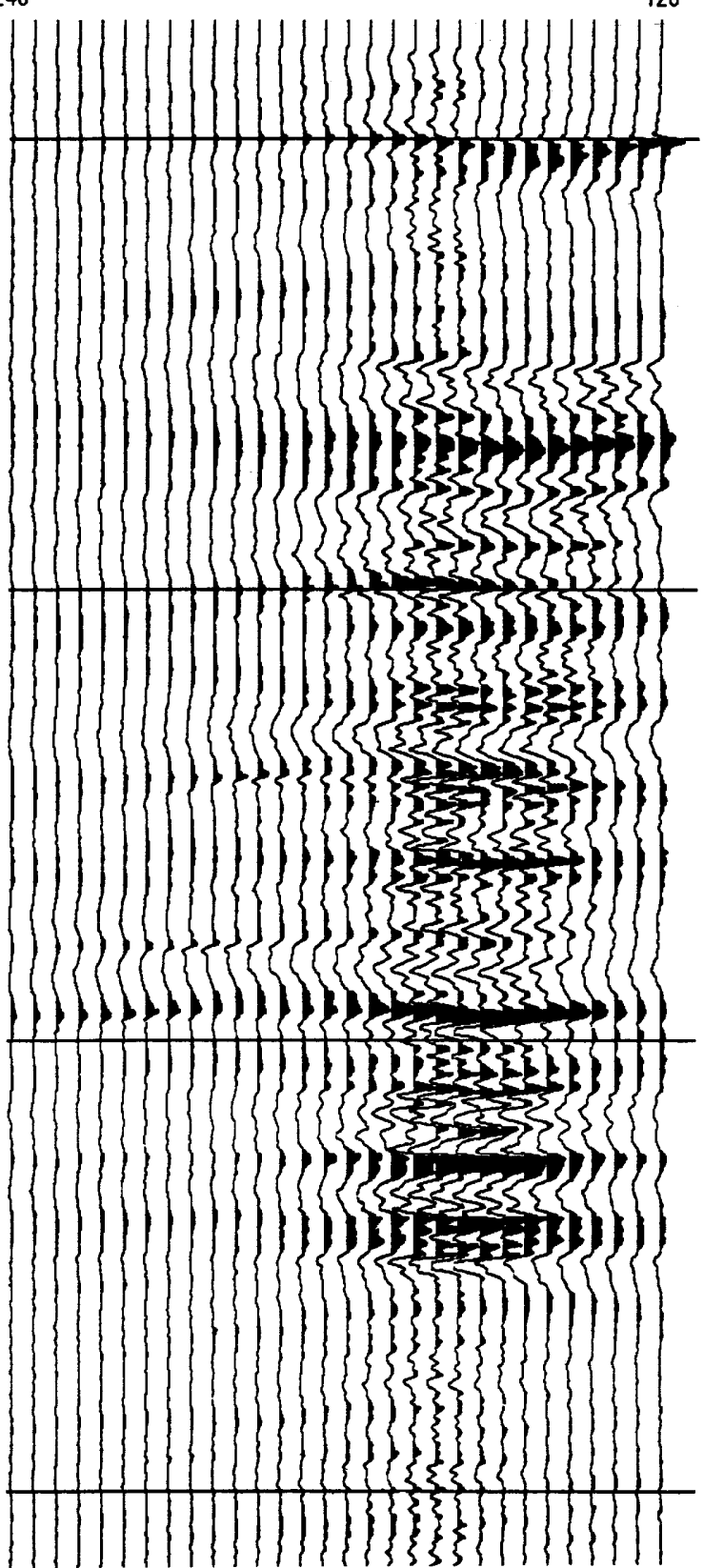

$\mathbf{B}$

Figure 19. Example of velocity analysis for a noise model. The noise model was generated by adding random-amplitude noise and static shifts to the noise-free model shown in figure $5 B$. Primary signal-to-random-noise ratio is about 2 , and the range of static shifts is $\pm 4 \mathrm{~ms}$. The range of moveout time at the far offset is between $-240 \mathrm{~ms}$ and $120 \mathrm{~ms}$, with a 12 -ms interval. The correction velocities for NMO to approximate parabolic moveouts are linearly increasing velocities with $1,500 \mathrm{~m} / \mathrm{s}$ at $2,500 \mathrm{~ms}$ and $1,700 \mathrm{~m} / \mathrm{s}$ at $4,500 \mathrm{~ms}$. $A$, Inverse operator; $B$, Transpose operator. 

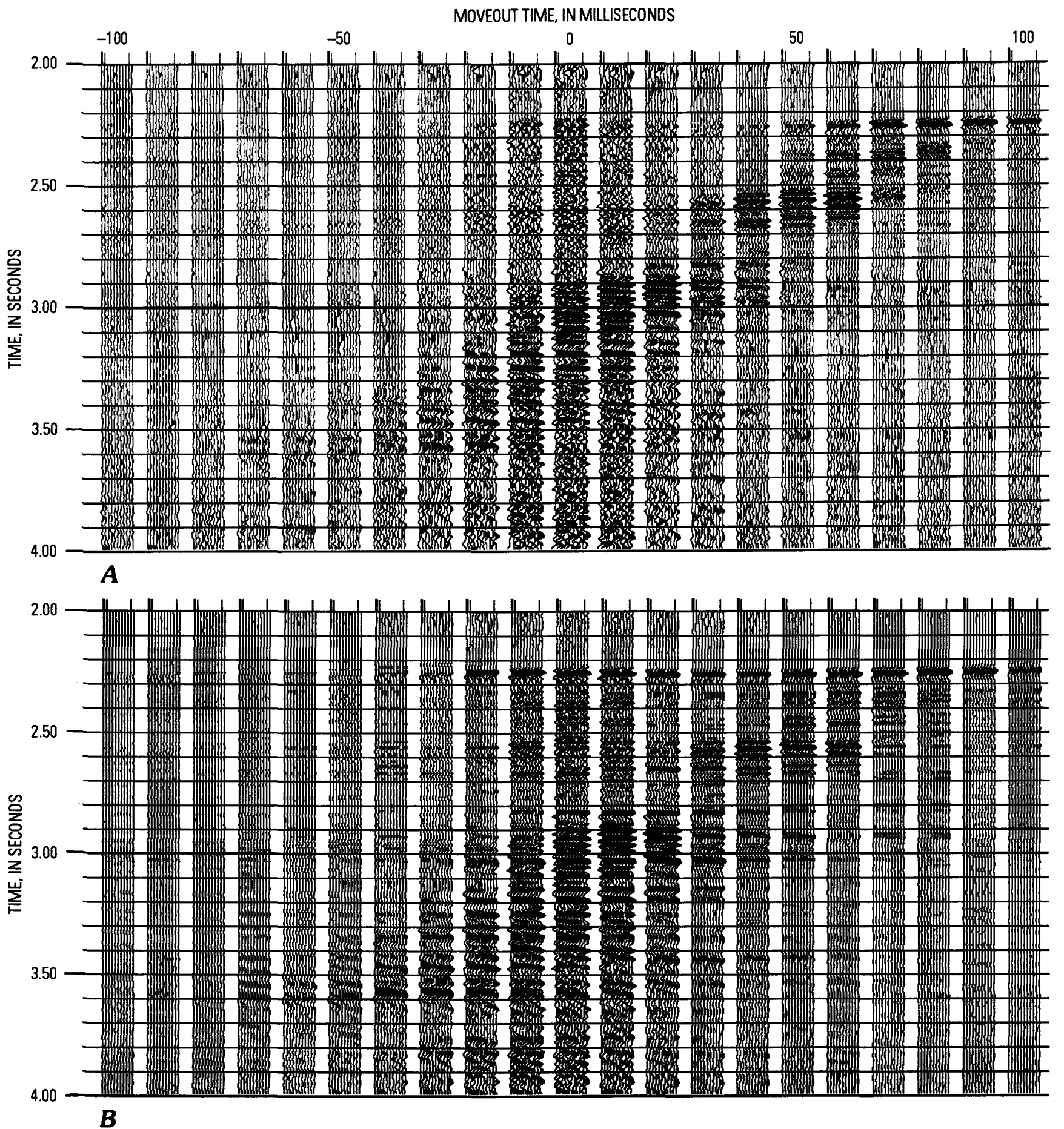

Figure 20. Example of velocity analysis for real data having 24 folds and near-offset distance of $400 \mathrm{~m}$ and far-offset distance of $2,777 \mathrm{~m}$. The constant correction velocity of $1,500 \mathrm{~m} / \mathrm{s}$ is used for NMO to approximate parabolic moveouts, and the range of moveout time at the far offset is between $-100 \mathrm{~ms}$ and $100 \mathrm{~ms}$, with a 10 -ms interval. $A$, Inverse operator; $B$, Transpose operator.

Figure 21 (facing page). Example of multiple suppression with and without static correction using an inverse operator. $A$, Straight stack; $B$, Multiple suppression without static correction; $C$, Multiple suppression with static correction. 


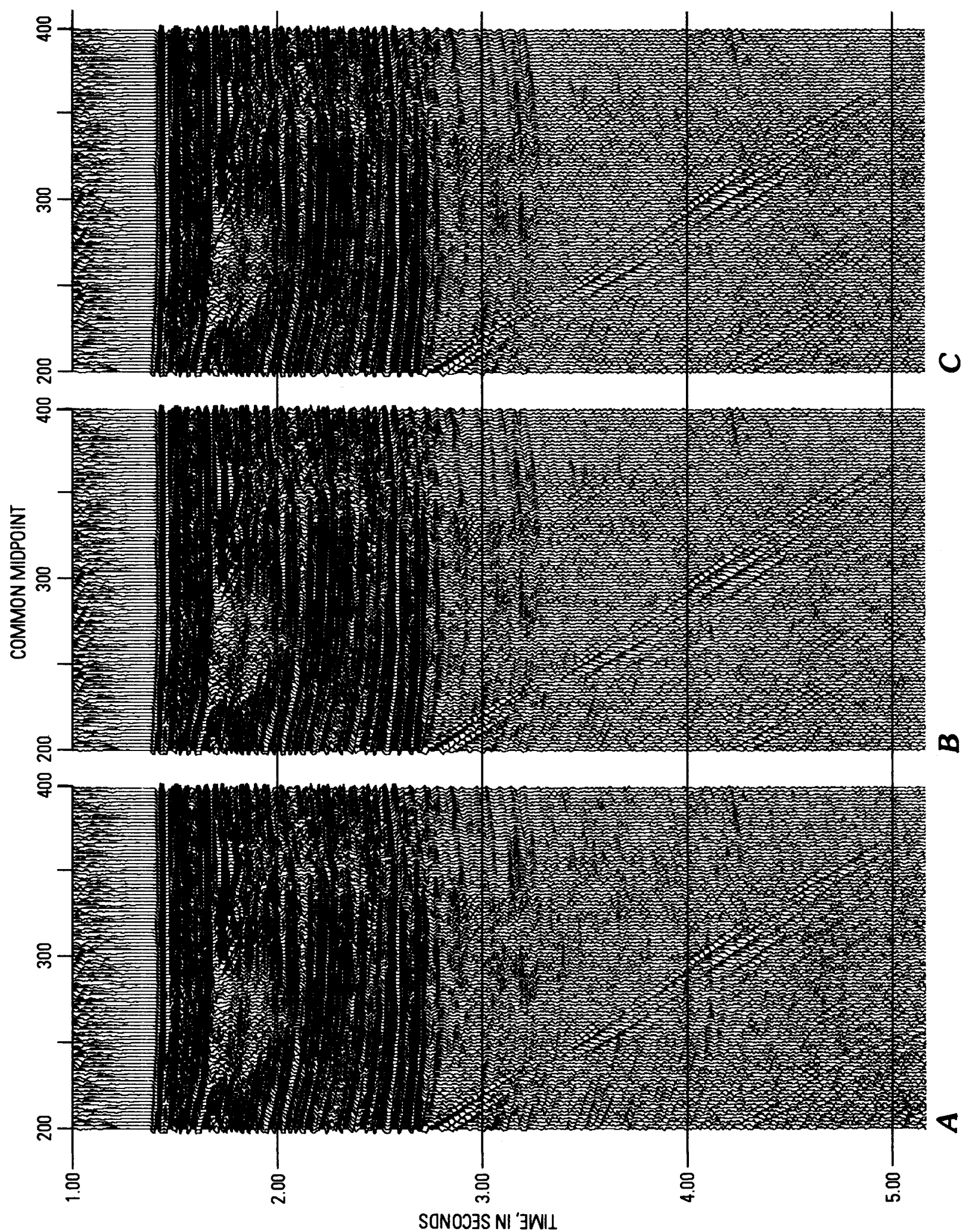




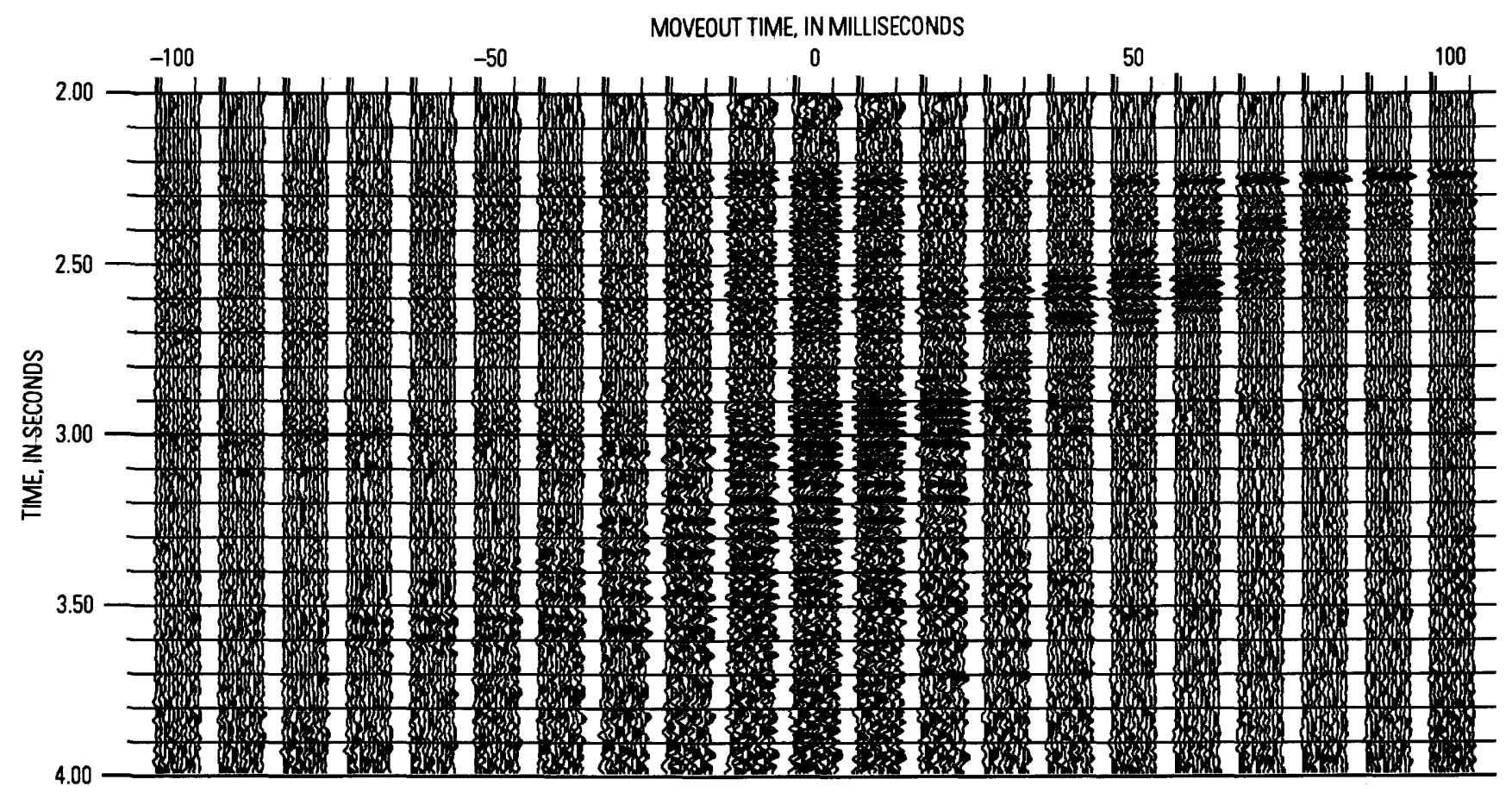

Figure 22. Plot showing the effect of random noise and static correction in a velocity analysis using the inverse operator. This figure is the same as figure $20 \mathrm{~A}$ except that (1) the amount of random noise in the inverse operator is 2 percent instead of 1 percent, as used in figure $20 \mathrm{~A}$, and (2) static correction was applied to the input data.

of the transpose operator with static corrections is not much different from the result shown in figure $20 B$. However, the result shown in figure 22 demonstrates that the improved performance of the inverse operator can be obtained with static corrections and by tuning the inverse operator with varied random noise.

\section{CONCLUSIONS}

A number of applications of velocity stack, such as multiple suppression, coherency filtering, trace interpolation, and velocity analysis, are described in this paper. The advantage of using an inverse operator over a transpose operator is in its ability to accurately estimate the model space from noisy seismic data. A transpose operator has a bias toward lower frequencies and more spread in the slowness domain. These properties result in trace mixing or smearing in the velocity stack. However, the advantage of using an inverse operator over the transpose operator decreases as noise increases. Based on this study, the following conclusions can be derived.

1. Because of the effectiveness of velocity stack at all offsets using an inverse operator, multiple suppression by velocity stack is better than the frequencywavenumber domain approach.
2. Because velocity stack relies on the alignment of seismic events along the moveouts, static correction of the CMP gathers is important in multiple suppression. Model studies indicate that about 4-ms static shifts in a gather degrades multiple suppression about $20 \mathrm{~dB}$.

3. Coherency filtering or trace interpolation by an inverse operator can be considered as a high-resolution method and minimizes trace mixing inherent to output by the transpose operator.

4. Velocity analysis by an inverse operator shows high discrimination ability of close velocities, but the amount of noise, such as static shifts, controls the performance rather dramatically. To obtain high-resolution velocity analysis for field data, pre-processing, such as static correction and amplitude adjustment, should be applied.

\section{REFERENCES CITED}

Aki, K., and Richards, P.G., 1980, Quantitative Seismology, Theory and Methods: San Francisco, W.H. Freeman Company, 911 p.

Barden, V., 1987, Trace interpolation in seismic data processing: Geophysical Prospecting, v. 35, p. 343-358.

Claerbout, J.F., 1984, Imaging the Earth's Interior: Blackwell Scientific Publications, 398 p. 
1992, Earth Sounding Analysis, Processing Versus Inversion: Blackwell Scientific Publications, 304 p.

Geist, E.L., Hart, P.E., and Fisher, M.A., 1990, A comparison of multiple suppression methods across a continental slope: Eos, Transactions, American Geophysical Union, v. 71, p. 1589.

Hampson, Dan, 1986, Inverse velocity stacking for multiple elimination: Journal of the Canadian Society of Exploration Geophysicists, v. 22, p. 44-55.

Kong, S.M., Phinney, R.A., and Roy-Chowdhury K., 1985, A nonlinear signal detector for enhancement of noisy seismic record sections: Geophysics, v. 50, p, 539-550.

Larner, K., Gibson, B., and Rothman, D., 1981, Trace interpolation and the design of seismic surveys [abs.]: Geophysics, v. 46, p. 407.

Monk, D.J., 1992, Approach to optimum slant stack and its application as a seismic noise attenuator [expanded abs.]: Society of Exploration Geophysicists, 62nd Annual International Meeting and Exposition, p. 1161-1164.

Published in the Central Region, Denver, Colorado Manuscript approved for publication May 26, 1994 Edited by Richard W. Scott, Jr.

Graphics prepared by Gayle Dumonceaux

Photocompositon by Carol Quesenberry
Ryu, J.V., 1982, Decomposition (DECOM) approach applied to wave field analysis with seismic reflection records: Geophysics, v. 47 , p. $869-883$.

Spitz, S., 1991, Seismic trace interpolation in the F-X domain: Geophysics, v. 56, p. 785-794.

Stoffa, P.L., Diebold, B.P., and Wenzel, F., 1981, Direct mapping of seismic data to the domain of intercept time and ray parameter-A plane wave decomposition: Geophysics, 46, p. 255-267.

Taner, M.T., and Koehler, F., 1969, Velocity spectra-Digital computer derivation and applications of velocity function: Geophysics, v. 34, 859-881.

Thorson, J.R., 1984, Velocity stack and slant stack inversion methods: Palo Alto, Calif., Stanford University, Ph.D. thesis.

Thorson, J.R., and Claerbout, J.F., 1985, Velocity stack and slant stack stochastic inversion: Geophysics, v. 50. p. 2727-2741. 





\section{Periodicals}

Earthquakes \& Volcanoes (issued bimonthly).

Preliminary Determination of Epicenters (issued monthly).

\section{Technical Books and Reports}

Professional Papers are mainly comprehensive scientific reports of wide and lasting interest and importance to professional scientists and engineers. Included are reports on the results of resource studies and of topographic, hydrologic, and geologic investigations. They also include collections of related papers addressing different aspects of a single scientific topic.

Bulletins contain significant data and interpretations that are of lasting scientific interest but are generally more limited in scope or geographic coverage than Professional Papers. They include the results of resource studies and of geologic and topographic investigations; as well as collections of short papers related to a specific topic.

Water-Supply Papers are comprehensive reports that present significant interpretive results of hydrologic investigations of wide interest to professional geologists, hydrologists, and engineers. The series covers investigations in all phases of hydrology, including hydrology, availability of water, quality of water, and use of water.

Circulars present administrative information or important scientific information of wide popular interest in a format designed for distribution at no cost to the public. Information is usually of short-term interest.

Water-Resources Investigations Reports are papers of an interpretive nature made available to the public outside the formal USGS publications series. Copies are reproduced on request unlike formal USGS publications, and they are also available for public inspection at depositories indicated in USGS catalogs.

Open-File Reports include unpublished manuscript reports, maps, and other material that are made available for public consultation at depositories. They are a nonpermanent form of publication that may be cited in other publications as sources of information.

\section{Maps}

Geologic Quadrangle Maps are multicolor geologic maps on topographic bases in $71 / 2$ - or 15 -minute quadrangle formats (scales mainly $1: 24,000$ or $1: 62,500$ ) showing bedrock, surficial, or engineering geology. Maps generally include brief texts; some maps include structure and columnar sections only.

Geophysical Investigations Maps are on topographic or planimetric bases at various scales, they show results of surveys using geophysical techniques, such as gravity, magnetic, seismic, or radioactivity, which reflect subsurface structures that are of economic or geologic significance. Many maps include correlations with the geology.

Miscellaneous Investigations Series Maps are on planimetric or topographic bases of regular and irregular areas at various scales; they present a wide variety of format and subject matter. The series also includes 7 1/2-minute quadrangle photogeologic maps on planimetric bases which show geology as interpreted from aerial photographs. The series also includes maps of Mars and the Moon.
Coal Investigations Maps are geologic maps on topographic or planimetric bases at various scales showing bedrock or surficial geology, stratigraphy, and structural relations in certain coal-resource areas.

Oil and Gas Investigations Charts show stratigraphic information for certain oil and gas fields and other areas having petroleum potential.

Miscellaneous Field Studies Maps are multicolor or black-andwhite maps on topographic or planimetric bases on quadrangle or irregular areas at various scales. Pre-1971 maps show bedrock geology in relation to specific mining or mineral-deposit problems; post-1971 maps are primarily black-and-white maps on various subjects such as environmental studies or wilderness mineral investigations.

Hydrologic Investigations Atlases are multicolored or black-andwhite maps on topographic or planimetric bases presenting a wide range of geohydrologic data of both regular and irregular areas; the principal scale is $1: 24,000$, and regional studies are at 1:250,000 scale or smaller.

\section{Catalogs}

Permanent catalogs, as well as some others, giving comprehensive listings of U.S. Geological Survey publications are available under the conditions indicated below from USGS Map Distribution, Box 25286, Building 810, Denver Federal Center, Denver, CO 80225. (See latest Price and Availability List.)

"Publications of the Geological Survey, 1879-1961" may be purchased by mail and over the counter in paperback book form and as a set microfiche.

"Publications of the Geological Survey, 1962-1970" may be purchased by mail and over the counter in paperback book form and as a set of microfiche.

"Publications of the U.S. Geological Survey, 1971-1981" may be purchased by mail and over the counter in paperback book form (two volumes, publications listing and index) and as a set of microfiche.

Supplements for 1982,1983,1984, 1985, 1986, and for subsequent years since the last permanent catalog may be purchased by mail and over the counter in paperback book form.

State catalogs, "List of U.S. Geological Survey Geologic and Water-Supply Reports and Maps For (State)," may be purchased by mail and over the counter in paperback booklet form only.

"Price and Availability List of U.S. Geological Survey Publications," issued annually, is available free of charge in paperback booklet form only.

Selected copies of a monthly catalog "New Publications of the U.S. Geological Survey" is available free of charge by mail or may be obtained over the counter in paperback booklet form only. Those wishing a free subscription to the monthly catalog "New Publications of the U.S. Geological Survey" should write to the U.S. Geological Survey, 582 National Center, Reston, VA 22092.

Note.-Prices of Government publications listed in older catalogs, announcements, and publications may be incorrect. Therefore, the prices charged may differ from the prices in catalogs, announcements, and publications. 


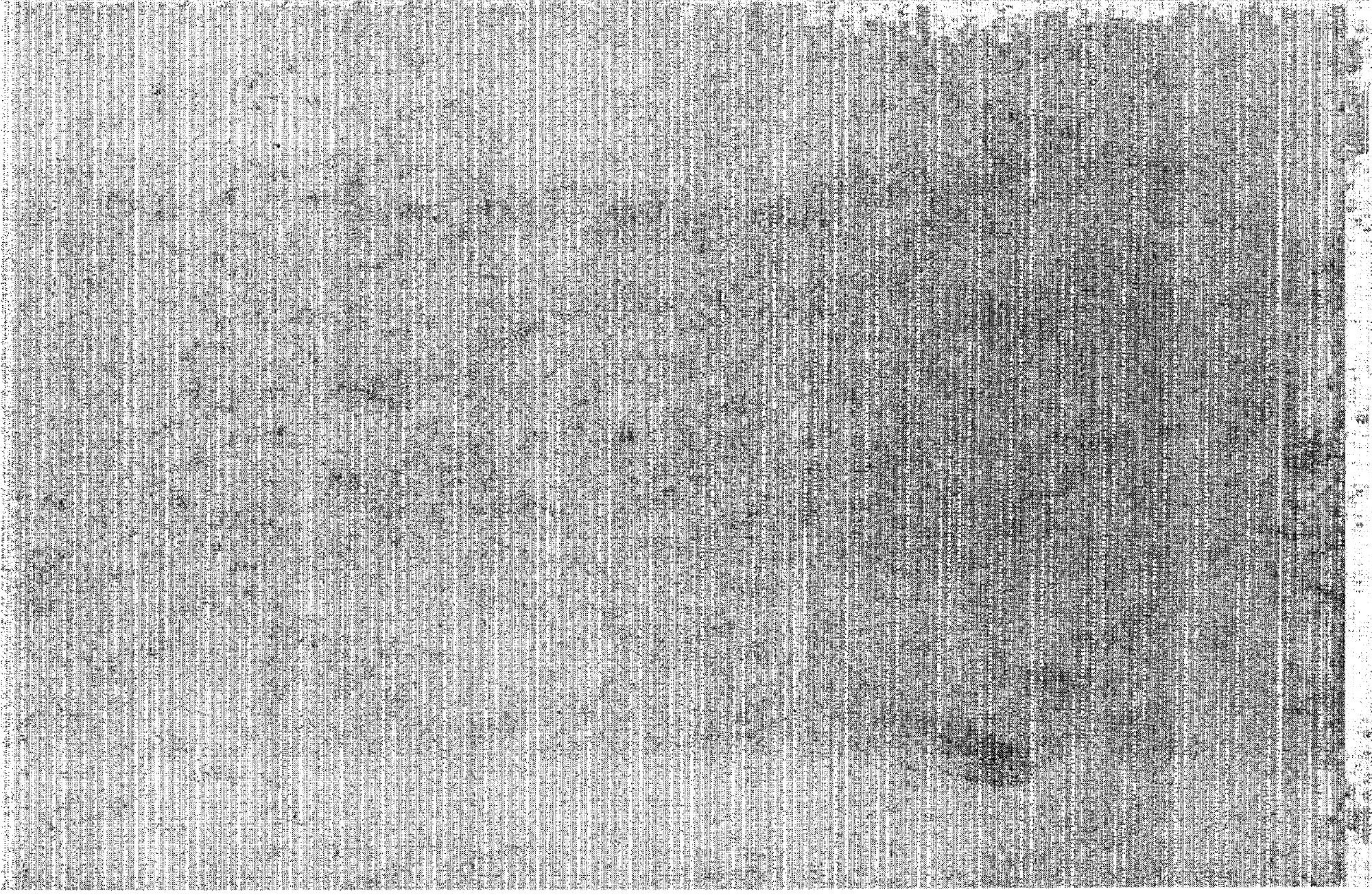

